\title{
A multivalent fuzzy interface drives reversible COPII coat assembly.
}

Viktoriya G. Stancheva ${ }^{1}$, Joshua Hutchings ${ }^{2}$, Xiao-Han Li ${ }^{1}$, Balaji Santhanam ${ }^{1}$, M. Madan Babu $^{1}$, Giulia Zanetti ${ }^{2}$, Elizabeth A. Miller ${ }^{1 *}$.

${ }^{1}$ MRC Laboratory of Molecular Biology

${ }^{2}$ Institute of Structural and Molecular Biology, Birkbeck College

*correspondence: emiller@mrc-Imb.cam.ac.uk

Abstract.

Protein secretion is initiated at the endoplasmic reticulum by the COPII coat, which selfassembles to form vesicles. Here, we examine the mechanisms by which the outer scaffolding layer of the coat drives local assembly of a structure rigid enough to enforce membrane curvature, yet able to readily disassemble at the Golgi. An intrinsically disordered region in the outer coat protein, Sec31, drives binding with an inner coat layer via multiple distinct interfaces. Interactions are individually dispensable but combinatorially reinforce each other, suggesting coat oligomerization is driven by the cumulative effects of multivalent interactions. Such a multimodal assembly platform could be readily reversed at the Golgi via perturbation of each individual interface. These design principles provide an explanation for how cells build a powerful yet transient scaffold to direct vesicle traffic.

\section{Introduction}

Eukaryotic cells secrete soluble and membrane-bound proteins to serve a variety of essential functions, including nutrient and ion uptake, cell-cell communication, and environmental signalling. Protein transport within the secretory pathway is facilitated by vesicles that are generated by cytoplasmic coat proteins, which simultaneously recruit appropriate cargo and sculpt the donor membrane into spherical structures. Membrane bending requires significant force to overcome the intrinsic rigidity of the lipid bilayer, and coat proteins solve this problem by self-organizing into oligomeric scaffolds that can impose structure on the underlying membrane. The coordination of coat polymerization with cargo 
recruitment is essential to avoid dead-end formation of empty vesicles, but inclusion of cargo can further increase the membrane bending energy required by the coat $(1,2)$. The paradox of such coat scaffolding oligomers is that the resultant assembly must be rigid enough to exert significant force, but also fully reversible to allow uncoating and vesicle fusion. In clathrin-mediated endocytosis, force generation is driven in part by the highly interdigitated clathrin triskelion, which requires ATP hydrolysis to disassemble. In contrast, the COPII and COPI coats, which mediate traffic in the early secretory pathway, form simpler scaffolds that can spontaneously disassemble. Here, we sought to address two aspects of COPII coat function: how coat assembly builds dynamic yet stable structures, and how such structures are reversed to prime fusion with the Golgi.

The COPII coat comprises five proteins that self-assemble on the cytosolic face of the endoplasmic reticulum (ER) membrane to drive traffic of nascent secretory proteins (Fig. 1A). COPII assembly is initiated upon GTP binding by the small GTPase, Sar1, which exposes an amphipathic $\alpha$-helix that embeds shallowly in the membrane (3). GTP-bound Sar1 recruits Sec23/Sec24 (4), which is the cargo-binding subunit of the coat $(5,6)$. The Sar1/Sec23/Sec24 "inner coat" complex in turn recruits the "outer coat", Sec13/Sec31, to drive vesicle formation $(4,7)$. Sec13/Sec31 tetramers form rods (8) that can self-assemble in vitro into a cage-like structure (Fig. S1A) that closely matches the size and geometry of vesicles observed by EM (9). Sar1/Sec23/Sec24 can also form higher order assemblies (10), but Sec13/Sec31 is required for organization of these arrays (3). Binding of the inner coat stimulates GTP hydrolysis on Sar1, and the outer coat further accelerates this reaction. The GTP hydrolysis cycle is key to the dynamic assembly and disassembly of the coat, thereby contributing to a dynamically metastable structure. Although the structures of individual coat layers are well-described, how they assemble locally to create rigid structures remains unclear. Moreover, accessory factors that are not integral to the coat, such as Sec16, also facilitate coat assembly via poorly defined mechanisms. Finally, how these stable oligomers are released from the membrane, both to recycle the coat proteins and to expose vesicle fusion machinery, is unknown (Fig. 1A).

In this study, we aimed to dissect the COPII coat assembly pathway by testing the functional importance of interfaces that drive outer coat recruitment to the inner coat layer and thus 
trigger membrane scaffolding. Two interactions between the inner and outer coat layers have been characterized biochemically and structurally $(11,12)$, both of which involve a long, unstructured domain of Sec31 (Fig. S1B). A short "active fragment" of Sec31 lies across the membrane-distal surface of Sec23/Sar1, contacting both proteins and stimulating Sar1

5 GTPase activity via two key residues, $W_{922}$ and $N_{923}$ in S. cerevisiae Sec31 (Fig. S1C) (11). A second mode of interaction involves triple-proline (PPP) motifs on Sec31 that bind to the gelsolin domain of $\operatorname{Sec} 23(3,12)$ (Fig. 1B, S1B). Exactly how these inter-subunit interfaces contribute to coat polymerization remains unclear. Moreover, these interfaces likely constitute important platforms for regulatory accessory factors that might modulate the COPII coat during different stages of vesicular transport $(12,13)$. A number of PPPcontaining proteins interact with COPII, for example to aid assembly (Sec16), or to promote formation of large carriers for transport of bulky cargoes in mammals (TANGO1). Here, we combine genetic perturbation with in vitro reconstitution assays to test the essentiality of individual interactions and determine the precise stage at which specific mutants are defective. We find that coat assembly is driven by a combination of evolutionarily conserved multivalent interactions between the inner and outer coat layers that combinatorially reinforce each other. Moreover, outer coat oligomerization via a known Sec31-Sec31 structural interface reinforces these interactions, suggesting a feed-forward mechanism for coat propagation. Our data define an assembly pathway that can be modulated by regulatory factors to confer reversibility to a metastable oligomeric structure without the input of energy to drive disassembly. Our findings also suggest a partial mechanism by which cells ensure directionality of vesicle traffic by local coat disassembly triggered at the Golgi by proteins competing for the same interaction interface.

PPP-driven interactions are dispensable for coat assembly but contribute to coat stability. We first tested the importance of PPP motifs in COPII assembly by mutagenesis of the Sec23 gelsolin domain that is known to interact with PPP motifs. Four critical aromatic residues that form the PPP-binding cleft are conserved between yeast and humans (Fig. 1B), and mutation of these residues abrogated PPP binding to human Sec23A (12). We engineered a

30 mutant that replaced key hydrophobic residues in the PPP-binding cleft with a glycineserine-glycine tripeptide. This gelsolin loop mutant (sec23- $\Delta g$ el) complemented a $\sec 23 \Delta$ null strain, revealing that PPP-binding is not essential for coat function (Fig. 1C). We next 
tested whether perturbations to the GTPase cycle of the coat might sensitize yeast to the loss of this PPP-binding interface. Sed4 is a non-essential accessory factor that is thought to assist Sec16 in Sar1 GTP regulation $(13,14)$. Indeed, sec23- $\Delta g e l$ was inviable when SED4 was also deleted, suggesting that compromised PPP-binding by Sec23 becomes problematic when the GTP cycle of the coat is altered (Fig. 1C). To gain further insight into the nature of the defect associated with perturbation of the gelsolin domain, we used an in vitro assay that reconstitutes vesicle formation from purified microsomal membranes (15). Vesicle release is monitored by the presence of cargo proteins, Erv46 and Sec22, in a slowly sedimenting vesicle fraction following incubation with purified COPII proteins. This budding assay showed that Sec23- $\Delta$ gel could drive vesicle formation with GMP-PNP but not with GTP (Fig. 1D). Thus, when coat assembly is stabilized by a non-hydrolysable GTP analog, perturbation of the gelsolin-PPP interaction has minimal effect, but under the condition of GTP-dependent coat turnover (7), loss of this interface impairs vesicle formation.

A GTP-specific vesicle budding defect was reminiscent of that observed with $\mathrm{N}$-terminally tagged Sec31, where the histidine tag is thought to interfere with assembly of the cage "vertex" interactions (Fig. S1A) that drive outer coat oligomerization (3). In the context of coat turnover promoted by GTP hydrolysis, further destabilization of the coat at cage vertices is incompatible with full coat assembly (3). We therefore tested the Sec23- $\Delta$ gel mutant in the budding assay in the presence of Nhis-Sec31, finding that vesicles fail to form even with GMP-PNP to stabilize the coat (Fig. 1E). Consistent with this, in a membrane bending assay, Sec23- $\Delta$ gel promoted tubulation of giant unilamellar vesicles (GUVs) into straight lattice-coated tubules in the presence of GMP-PNP and wild type Sec31. However, when GUV tubulation was induced using Sec23- $\Delta$ gel and Nhis-Sec31, tubes were irregular and no extended coat lattice could be visualised (Fig. 1F). One interpretation of these findings is that two separate interactions - Sec23/Sec31 binding via PPP motifs and cage assembly via Sec31/Sec31 vertex interfaces - mutually reinforce each other to stabilize the coat and propagate coat assembly. Such a feed-forward loop to drive vesicle formation might prevent inappropriate assembly away from the ER membrane. These mutually stabilizing interactions are especially important under conditions of GTP hydrolysis, where the coat is inherently unstable (7). Our Sec23 mutagenesis experiments suggest that PPP 
binding contributes to but is not essential for coat assembly. The importance of the PPP interaction is revealed when the GTP cycle of the coat is perturbed, suggesting that the nucleotide-associated interface and PPP binding are mutually productive in COPII assembly.

5 We next sought to test the importance of specific PPP motifs by mutagenesis of Sec31, additionally abrogating the "active fragment" that also contributes to inner-outer coat binding (Fig. 2A). Within the active fragment, residues $\mathrm{W}_{922}$ and $\mathrm{N}_{923}$ drive GTPase stimulation (11) and are likely the most stable part of the interaction (3). Alanine substitution of these amino acids had no effect on cell viability (Fig. 2B). Similarly,

10 glycine/serine substitution of all 6 PPP motifs, plus an additional PPAP motif that might also bind to the gelsolin domain (12) (sec31- $\triangle P P P)$ caused no growth defect, even when combined with the catalytic $\mathrm{W}_{922} \mathrm{~A} / \mathrm{N}_{923} \mathrm{~A}$ mutation (Fig. $2 \mathrm{~B}$ ). In a coat recruitment assay that measures binding of purified proteins to large (400nm) unilamellar liposomes in the presence of GMP-PNP, all variant proteins were recruited normally (Fig. S2A). Moreover, when the different mutants were used in microsomal budding reactions, each protein supported vesicle formation both with GTP and GMP-PNP, although vesicle formation was reduced with the combined $\mathrm{W}_{922} \mathrm{~A} / \mathrm{N}_{923} \mathrm{~A} / \Delta \mathrm{PPP}$ mutant (Fig. $2 \mathrm{C}$ ). We tested whether destabilization of cage interfaces by the introduction of the N-terminal His tag would sensitize these mutants as it had for the sec23- $\Delta$ gel mutant. Although Nhis-Sec31 supports viability, in this background the $\triangle P P P$ and $W_{922} \mathrm{~A} / \mathrm{N}_{923} \mathrm{~A} / \triangle \mathrm{PPP}$ mutants were inviable (Fig. 2D). Nonetheless, these mutant proteins were competent in the coat recruitment (Fig. 2E, Fig. S2B) and tubulation assays (Fig. 2F). Thus, the known interaction motifs cannot explain the entirety of inner coat/outer coat assembly, nor of inner coat array formation.

A charge-driven interaction interface contributes to coat assembly.

Since the catalytic and PPP motifs didn't fully account for inner/outer coat interactions, we examined the Sec31 disordered region for additional features that might contribute to coat assembly (16). We calculated charge properties across Sec31, focusing on the fraction of charged residues and net charge per residue. We found extensively charged clusters of residues across different regions of $\operatorname{Sec} 31$, including clusters of net negatively charged residues in the structured domain, and smaller clusters of net positive charges in the 
disordered region (Fig. 3A). These positive charge clusters were also features of Sec31 orthologues in human and Arabidopsis (Fig. S3A), suggestive of an evolutionarily conserved property. We searched for a corresponding surface on Sec23 that may comprise a chargedriven interaction interface between the inner and outer coats. We identified a negatively charged surface adjacent to the gelsolin domain, separate from that occupied by the "active fragment" (Fig. 3B, top panel). Reversing the charges of this region on Sec23 (Fig. 3B, bottom panel) led to lethality (Fig. 3C), and the purified protein was defective for recruitment of Sec13/Sec31 in the liposome binding assay (Fig. 3D). We propose that charge-based interactions between Sec23 and Sec31 represent a third binding mode that contributes to inner/outer coat interactions (Fig. 3E(i)). We note that three different types of interaction drive association of the inner and outer coats: a nucleotide-driven interface mediated by $\mathrm{W} / \mathrm{N}$ residues, linear (PPP) motifs, and a charge-driven interaction. Whether the W/N, PPP and charge interactions drive binding to the same Sec23 molecule, or instead bridge adjacent $\operatorname{Sec} 23 / \mathrm{Sec} 24$ complexes to contribute to inner coat assembly remains to be 15 determined (Fig. 3E(i)).

\section{Disordered region-driven competition can contribute to reversible coat interactions.} Using mechanistically distinct interfaces to build a global set of interactions might contribute robustness to the system while maintaining reversibility by different mechanisms: GTP hydrolysis to weaken the catalytic interface, phosphorylation of the charged regions to reverse binding, and competition from other PPP-containing proteins to disrupt the interaction interface (12). We sought proof of principle that a Golgi-localized PPP-containing protein might help perturb coat structure by capitalizing on the dynamic nature of an interaction interface driven by a disordered region (17). Grh1 is a GRASPdomain protein that together with Uso1 and Bug1 forms a COPII tethering complex that interacts with Sec23/Sec24 $(18,19)$. The C-terminal domain of Grh1 is predicted to be disordered, with a net positively charged cluster, and a PPP motif (Fig. S4). Purified Grh1 competed for Sec31 binding to immobilized GST-Sec23, and deletion of its disordered region abrogated this effect (Fig. 3F). This observation suggests that weak PPP- and charge-driven

30 interactions modulate coat assembly and disassembly at distinct spatial localizations (Fig. $3 E(i i))$. 


\section{Coat robustness stems from combinations of interaction interfaces.}

Having identified multiple modes of interaction across the Sec31 disordered region, we sought to better define the importance and hierarchy of the different Sec23-binding elements. We divided the Sec31 disordered region into three segments of equivalent length and generated constructs that preserved the structural domains (the $\mathrm{N}$-terminal $\beta$-propeller that forms the cage vertex, the $\alpha$-solenoid rods and a C-terminal $\alpha$-solenoid domain) but replaced the disordered region with different non-overlapping shortened fragments. Each segment retained at least one PPP motif and two positive-charge clusters (Fig. 4A). The first third $\left(\sec 31_{A}\right)$, with a single PPP motif and two charge clusters, was unable to support viability, whereas each of the two subsequent segments $\left(\sec 31_{B}\right.$ and $\left.\sec 31_{C}\right)$, which both contained multiple PPP motifs and charge clusters, complemented a sec31 $\Delta$ null strain (Fig. 4A). We note that deletion of all PPP motifs in full-length Sec31, where $\mathrm{W} / \mathrm{N}$ and charge patches remain, was not lethal (Fig. 2B). In contrast, deletion of the four PPP motifs in Sec31c, which lacks the $\mathrm{W} / \mathrm{N}$ interaction but retains charge patches, abrogated viability (Fig. $\left.4 \mathrm{~B}, \sec 31_{C}-\triangle P P P\right)$. This lethality suggests that PPP motifs become more important in the context of a shorter fragment that presumably has less overall affinity for Sec23. We further dissected the C-terminal portion of the disordered region into two smaller fragments, each of which contained at least two PPP motifs and one charge cluster, but neither supported viability ( $\sec 31_{D}$ and $\sec 31_{E}$, Fig. 4A). We tested each of the shortened constructs in the liposome binding assay, observing that proteins that conferred viability also supported coat recruitment (Fig. 4C). However, in the in vitro budding assay, we saw distinct effects for Sec31 $1_{B}$ and Sec31 (Fig. 4D). Budding reactions with GTP were supported by Sec31 but not $\operatorname{Sec} 31_{B}$, consistent with the active fragment contained within $S e c 31_{B}$ stimulating GTP hydrolysis and thereby destabilizing the coat prematurely. Conversely, in the presence of GMP-PNP, Sec31 $1_{B}$ supported vesicle release whereas Sec31 was less effective. Again, this is consistent with the known interface occupied by the active fragment, where the Sec23/Sar1•GMP-PNP complex would stabilize association with Sec31 , prolonging interaction to drive vesicle formation.

30 The in vitro and in vivo phenotypes of these minimal constructs highlight the different assembly elements: the $\mathrm{W} / \mathrm{N}$ motif that provides nucleotide-dependent affinity; linear 
motifs (PPP) that form weak but specific binding interfaces; a charge-driven interface; multivalency of these weak interactions; and disordered region length. The absence of the $\mathrm{W} / \mathrm{N}$ interface can be compensated for by four PPP motifs and charge patches (Sec31c), but not by charges in the context of a single PPP $\left(\operatorname{Sec} 31_{A}\right)$. Moreover, three PPP motifs with charge patches in the context of a short fragment (Sec31 $\mathrm{E}$ ) cannot support assembly. Such compensation suggests that the different interfaces are partially redundant but reinforce each other to make a robust system. Orthologous Sec31 proteins across species may alter how they employ these interfaces to create functional proteins that might exhibit distinct assembly properties.

Coat assembly can be driven by diverse sequences with conserved properties.

Our charge/disorder analysis revealed conserved properties within the Sec31 disordered regions across species even though primary sequence identity was relatively low (Fig. S3B). This is in contrast to sequence conservation across the structured domains, which is

15 relatively high (Fig. S3B), and the purified proteins show clear structural similarity. We reasoned that if coat assembly is driven by a combination of multi-modal interactions rather than protein sequence properties, a disordered region from an orthologous Sec31 might functionally replace the yeast domain. We created such chimeric proteins where the yeast disordered region was replaced with that of human Sec31A (23\% similarity to the yeast

20 Sec31 disordered region), human Sec31B (26\% similarity) or Arabidopsis Sec31A (15\% similarity) (Fig. 5A). Each of the substitute disordered regions, although differing in length, contained clusters of positively charged residues (Fig. S3A). Each orthologue had two PPP (or PPAP/PAPP) motifs, but both human Sec31B and Arabidopsis Sec31A lacked the catalytic $\mathrm{W} / \mathrm{N}$ residues. When these constructs were introduced into yeast, only the chimera with the disordered region of $\mathrm{HsSec} 31 \mathrm{~A}$ disordered region ( $\left.\sec 31-H s 31 A_{D R}\right)$ supported viability as the sole copy of Sec31 (Fig. 5A). Abrogation of the PPP motifs in this construct reduced viability (Fig. 5B), confirming that these linear motifs are indeed important in driving coat assembly. We tested the two yeast-human chimeric proteins in liposome recruitment (Fig. 5C) and budding assays (Fig. 5D), finding activity mirrored the in vivo phenotypes. Only Sec31-

$30 \mathrm{Hs} 31 \mathrm{~A}_{D R}$ was able to bind Sar1/Sec23/Sec24 and to drive budding from microsomal membranes. We note that the two disordered regions that were non-functional in yeast, $\mathrm{HsSec} 31 \mathrm{~B}$ and AtSec31A, both lack catalytic $\mathrm{W} / \mathrm{N}$ residues, suggesting that one of the three 
modes of interaction is not preserved in these proteins. In both cases, these proteins are one of multiple Sec31 paralogs, suggesting that some species might employ mixed coats containing both catalytic and non-catalytic subunits. This might be a mechanism of modulating the global rate of GTPase activity, and thus coat turnover, to fine-tune coat assembly.

Given the surprising finding that a human disordered region can function in yeast despite such divergence ( $23 \%$ similarity) in protein sequence, we sought to test even more distant domains that share similar properties. We first asked whether the disordered regions of Sec16 could similarly replace that of Sec31. Sec16 localises to ER exit sites and is known to interact with Sec23 and Sec24, likely acting as a scaffold to initiate COPII recruitment and organisation (20). Sec16 has two intrinsically disordered regions that lie upstream and downstream of a conserved central domain (CCD), which is a structured helical region that interacts with Sec13 (21) (Fig. 6A). The upstream disordered region (DR1) interacts with

15 Sec24 (22) and contains a single PPP motif along with several positive charge clusters (Fig. $6 A$ ), whereas the downstream region (DR2) interacts with Sec23 (22), and contains multiple PPP motifs and significant areas of both net positive and negative charge (Fig. 6A). We replaced the Sec31 disordered region with Sec16 $6_{D R 1}$ or Sec16 $6_{D R 2}$ and tested each chimera for their ability to function in place of Sec31. Both Sec31-16DR1 and Sec31-16DR2 were recruited to the coat in the liposome binding assay (Fig. 6B), suggesting that each could interact with the inner coat to support initial assembly. In contrast, only Sec31-16 $6_{\text {DR } 1}$ could complement a sec31 $\Delta$ strain (Fig. 6C), and support budding from microsomal membranes, albeit less efficiently than wild-type Sec31 (Fig. 6D). Since Sec31-16 DR2 could bind to membranes but not support microsomal budding, we tested whether it could tubulate GUVs in vitro, and found this protein to be inactive. The discrepancy between coat binding by Sec31-16DR2 but failure to drive vesicle formation or support viability suggests that the mode of interaction driven by the second disordered region of Sec16 is not compatible with the specific coat conformation required for membrane bending. We note that Sec16 ${ }_{D R 2}$ has multiple features that may impact inner coat interactions, most notably significant negatively charged clusters that are not present in Sec31 disordered regions. This divergence is probably indicative of the distinct function that Sec16 serves as an accessory or regulatory factor. 
We next searched the yeast genome for additional proteins that have predicted disordered regions that also contain multiple PPP motifs. We observed that such proteins fell into three broad functional categories (Fig. S5A). The COPII category comprises Sec31 and the accessory protein, Sec16. A second category consists of proteins involved in endocytosis and actin regulation, and a third contains proteins involved in RNA processing (Fig. S5A). In a recent analysis of yeast disordered segments (23), several of the endocytosis/actin proteins had multiple properties (16) within disordered regions that were similar to Sec31 (Fig. S5B). We therefore tested whether some of these disordered regions could function in COPII coat assembly when replacing the endogenous Sec31 disordered region. Indeed, the disordered region of Las17 could functionally replace that of Sec31 to support viability (Fig. 6E). Las17 is an actin assembly factor that uses its proline rich domain to nucleate actin filaments (24). This mode of interaction is shared by other components of the actin/endocytic system and has parallels with the COPII coat in that multivalent weak interactions drive assembly (25). We note that the Las17 region we tested shares several key features with other regions that could function in the context of Sec31: multiple PPP motifs, clusters of net positive charge in the absence of significant net negative charge, and significant length. Since Las17 has no $\mathrm{W} / \mathrm{N}$ motif, clearly these features alone suffice to drive coat assembly, highlighting the plasticity of the inner/outer coat interaction.

20 Together, our mutagenesis and disordered region replacement experiments reveal a multimodal mechanism of coat assembly, where interactions between the inner and outer coat layers comprise a multivalent fuzzy interface $(26,27)$. Individual interactions derive from distinct elements that include linear motifs (PPP), charge (positive charge clusters), and length. Each individual interaction is dispensable, but in concert they drive function. The precise role of disordered region length remains to be explored; one possibility is that a shortened disordered region (for example the Sec31 fragment which still contains multiple interaction sites) fails to assemble because of an inability to bridge adjacent Sec23 complexes. A coat assembly/disassembly mechanism driven by fuzzy interactions provides a possible mechanistic explanation for how a metastable structure like a vesicle coat can form in a controlled manner (27). By building up from initially weak, transient interactions, the coat can be recruited locally but is not committed to full assembly until a threshold of both inner and outer coat components is reached. Moreover, upstream accessory factors, like 
Sec16 and TANGO1, that use the same interactions can prime an exit site for Sec31 recruitment and organize the inner coat. Once Sec31 is engaged, its disordered region can act as molecular velcro, being strong yet readily reversible by specific mechanisms. Coat propagation could feed forward as cage vertex interactions (mediated by the $\beta$-propeller domain of Sec31) bring in additional outer coat rods to in turn organize more inner coat complexes. As the diversity of Sec31 paralogs expands, as seen in multicellular organisms, disordered regions may fuel evolution of altered interfaces that allow for fine-tuning of vesicle assembly, disassembly and geometry (28). For instance, the lack of the catalytic W/N motif in human Sec31B may reflect the need for a more stable coat structure, whereby the GTPase activity of the coat is not stimulated. Such stability may prolong initial events during coat assembly to favour formation of non-canonical carriers.

The multiple assembled interfaces between Sec23 and Sec31 could contribute to persistence of the polymerized coat even after GTP hydrolysis by Sar1 and its release from the membrane. Evidence for preserved coat elements on Sar1-free vesicles comes from immunogold labelling experiments that revealed persistent Sec23 and Sec13 on COPII vesicles generated with GTP (15). Similarly, multivalent combinatorial interactions provide an excellent mechanism for how a metastable coat polymer can be disassembled without the need for uncoating chaperones that expend energy. A combinatorial binding mode allows a stable structure to "breathe" via dynamic weak individual interactions that permit an opposing similarly weak interaction to compete and destabilize the structure. In the case of the COPII coat, long-distance tethering by extended coiled-coil proteins, like Uso1, could capture a coated vesicle, which would be brought close to the Golgi membrane, where Grh1 could locally act to initiate release of Sec13/Sec31. Such a model of locally triggered uncoating, perhaps further stimulated by Golgi-localized kinases like Hrr25 (29), could help ensure the directionality of vesicle traffic. Our findings of coat assembly driven by interactions involving intrinsically disordered regions are also likely to apply to other vesicle trafficking steps. Each of the major coat complexes have significant disordered regions within their subunits (30), and the flexible nature of the clathrin light chain is important in coat assembly (31). Our approach and the findings that we present here provide a conceptual framework to now discover and investigate how such elements in disordered regions of other coat complexes are used for dynamic assembly and disassembly. 


\section{References}

1. J. C. Stachowiak, F. M. Brodsky, E. A. Miller, A cost-benefit analysis of the physical mechanisms of membrane curvature. Nat Cell Biol. 15, 1019-1027 (2013).

52 J. Derganc, B. Antonny, A. Copič, Membrane bending: the power of protein imbalance. Trends Biochem Sci. 38, 576-584 (2013).

3. J. Hutchings, V. Stancheva, E. A. Miller, G. Zanetti, Subtomogram averaging of COPII assemblies reveals how coat organization dictates membrane shape. Nature Communications. 9, 4154-8 (2018).

10 4. K. Matsuoka et al., COPII-coated vesicle formation reconstituted with purified coat proteins and chemically defined liposomes. Cell. 93, 263-275 (1998).

5. E. Mossessova, L. C. Bickford, J. Goldberg, SNARE selectivity of the COPII coat. Cell. 114, 483-495 (2003).

6. E. Miller, B. Antonny, S. Hamamoto, R. Schekman, Cargo selection into COPII vesicles is driven by the Sec24p subunit. EMBO J. 21, 6105-6113 (2002).

7. B. Antonny, D. Madden, S. Hamamoto, L. Orci, R. Schekman, Dynamics of the COPII coat with GTP and stable analogues. Nat Cell Biol. 3, 531-537 (2001).

8. S. Fath, J. D. Mancias, X. Bi, J. Goldberg, Structure and organization of coat proteins in the COPII cage. Cell. 129, 1325-1336 (2007).

S. M. Stagg et al., Structure of the Sec13/31 COPII coat cage. Nature. 439, 234-238 (2006).

10. G. Zanetti et al., The structure of the COPII transport-vesicle coat assembled on membranes. Elife. 2, e00951-e00951 (2013).

11. X. Bi, J. D. Mancias, J. Goldberg, Insights into COPII coat nucleation from the structure of Sec23.Sar1 complexed with the active fragment of Sec31. Dev Cell. 13, 635-645 (2007).

12. W. Ma, J. Goldberg, TANGO1/CTAGE5 receptor as a polyvalent template for assembly of large COPII coats. Proceedings of the National Academy of Sciences. 113, 10061-10066 (2016).

$30 \quad 13 . \quad$ L. F. Kung et al., Sec24p and Sec16p cooperate to regulate the GTP cycle of the COPII coat. EMBO J. 31, 1014-1027 (2012).

14. R. E. Gimeno, P. Espenshade, C. A. Kaiser, SED4 encodes a yeast endoplasmic reticulum protein that binds Sec16p and participates in vesicle formation. J Cell Biol. 131, 325-338 (1995). 
15. C. Barlowe et al., COPII: a membrane coat formed by Sec proteins that drive vesicle budding from the endoplasmic reticulum. Cell. 77, 895-907 (1994).

16. R. van der Lee et al., Classification of intrinsically disordered regions and proteins. Chem. Rev. 114, 6589-6631 (2014).

5 17. R. B. Berlow, H. J. Dyson, P. E. Wright, Hypersensitive termination of the hypoxic response by a disordered protein switch. Nature. 543, 447-451 (2017).

18. R. Behnia, F. A. Barr, J. J. Flanagan, C. Barlowe, S. Munro, The yeast orthologue of GRASP65 forms a complex with a coiled-coil protein that contributes to ER to Golgi traffic. J Cell Biol. 176, 255-261 (2007).

19. M. Schuldiner et al., Exploration of the Function and Organization of the Yeast Early Secretory Pathway through an Epistatic Miniarray Profile. Cell. 123, 507-519 (2005).

20. F. Supek, D. T. Madden, S. Hamamoto, L. Orci, R. Schekman, Sec16p potentiates the action of COPII proteins to bud transport vesicles. J Cell Biol. 158, 1029-1038 (2002).

21. J. R. R. Whittle, T. U. Schwartz, Structure of the Sec13-Sec16 edge element, a template for assembly of the COPII vesicle coat. J Cell Biol. 190, 347-361 (2010).

22. R. E. Gimeno, P. Espenshade, C. A. Kaiser, COPII coat subunit interactions: Sec24p and Sec23p bind to adjacent regions of Sec16p. Mol Biol Cell. 7, 1815-1823 (1996).

23. T. Zarin et al., Proteome-wide signatures of function in highly diverged intrinsically disordered regions. Elife. 8, 1727 (2019).

24. A. N. Urbanek, A. P. Smith, E. G. Allwood, W. I. Booth, K. R. Ayscough, A Novel Actin-Binding Motif in Las17/WASP Nucleates Actin Filaments Independently of Arp2/3. Curr Biol. 23, 196-203 (2013).

25 25. Y. Sun et al., Switch-like Arp2/3 activation upon WASP and WIP recruitment to an apparent threshold level by multivalent linker proteins in vivo. Elife. 6, 60 (2017).

26. M. Fuxreiter, Fuzziness in Protein Interactions-A Historical Perspective. Journal of Molecular Biology. 430, 2278-2287 (2018).

27. H. Wu, M. Fuxreiter, The Structure and Dynamics of Higher-Order Assemblies: Amyloids, Signalosomes, and Granules. Cell. 165, 1055-1066 (2016).

28. M. M. Babu, R. W. Kriwacki, R. V. Pappu, Structural biology. Versatility from protein disorder. Science. 337, 1460-1461 (2012).

29. C. Lord et al., Sequential interactions with Sec23 control the direction of vesicle traffic. Nature. 473, 181-186 (2011). 
30. N. Pietrosemoli, R. Pancsa, P. Tompa, Structural Disorder Provides Increased Adaptability for Vesicle Trafficking Pathways. PLoS Comput Biol. 9, e1003144-19 (2013).

31. J. D. Wilbur et al., Conformation switching of clathrin light chain regulates clathrin lattice assembly. Dev Cell. 18, 841-848 (2010).

32. Y. Shimoni, R. Schekman, Vesicle budding from endoplasmic reticulum. Meth Enzymol. 351, 258-278 (2002).

33. L. J. Wuestehube, R. S. M. I. enzymology, 1992, [13] Reconstitution of transport from endoplasmic reticulum to golgi complex using endoplasmic reticulumenriched membrane fraction from yeast. Elsevier

34. B. Mészáros, G. Erdős, Z. Dosztányi, IUPred2A: context-dependent prediction of protein disorder as a function of redox state and protein binding. Nucleic Acids Research. 46, W329-W337 (2018).

35. A. S. Holehouse, R. K. Das, J. N. Ahad, M. O. G. Richardson, R. V. Pappu, CIDER: Resources to Analyze Sequence-Ensemble Relationships of Intrinsically Disordered Proteins. Biophys J. 112, 16-21 (2017).

36. F. Madeira et al., The EMBL-EBI search and sequence analysis tools APIs in 2019. Nucleic Acids Research. 47, W636-W641 (2019).

37. R. Sikorski, P. Hieter, A system of shuttle vectors and yeast host strains designed for efficient manipulation of DNA in Saccharomyces cerevisiae. Genetics. 122, 1927 (1989).

\section{Acknowledgements}

We thank Wanda Kukulski and Manu Hegde for comments on the manuscript, Jianguo Shi

for help with insect cell culture, Mark McClintock for assistance with protein purification, and David Owen for advice on Sec23 mutagenesis. Funding: This work was supported by funding from the UK Medical Research Council (MRC_UP_1201/10 to EAM and MC_U105185859 to MMB); an AMS Springboard award (SBF0031030), the ERC StG (852915 - CRYTOCOP) and the BBSRC (BB/T002670/1) to GZ; and by an EMBO Marie Curie fellowship to XL. Author contributions: Conceptualization: EAM, MMB; Funding acquisition: EAM, GZ, $\mathrm{MMB}, \mathrm{XL}$; Investigation: VS, JH, GZ, XL, BS, EAM; Writing - original draft: EAM; Writing review and editing: VS, JH, GZ, XL, BS, MMB, EAM. Competing interests: The authors declare no competing interests. Data and materials availability: All data is available in the 
bioRxiv preprint doi: https://doi.org/10.1101/2020.04.15.043356; this version posted April 16, 2020. The copyright holder for this preprint (which was not certified by peer review) is the author/funder. All rights reserved. No reuse allowed without permission.

manuscript or the supplementary materials; plasmids and strains described can be obtained from EAM.

\section{Supplementary Materials:}

5 Materials and Methods

Figures S1 - S5

Tables S1 and S2 
Figures and legends:

A

ER

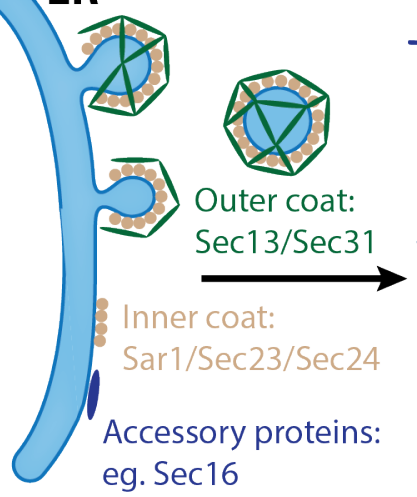

C

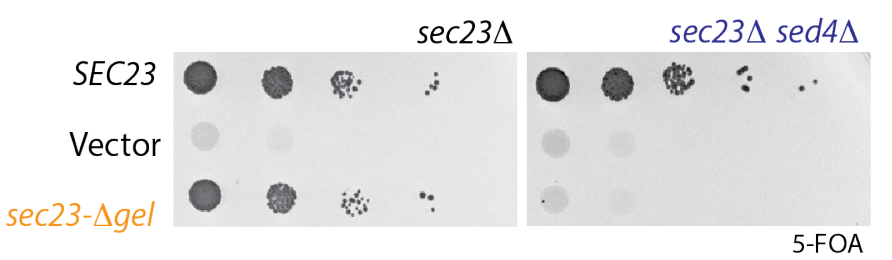

$\mathrm{E}$

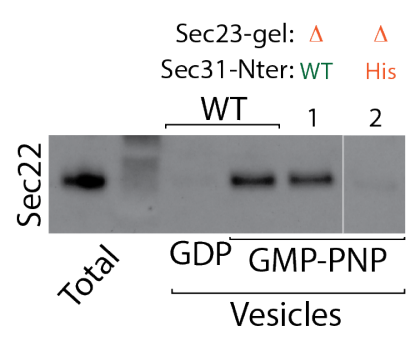

B

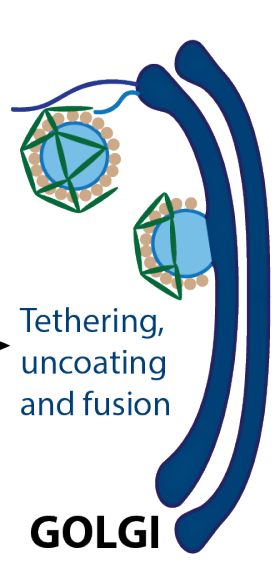

GOLGI
$\mathrm{F}$
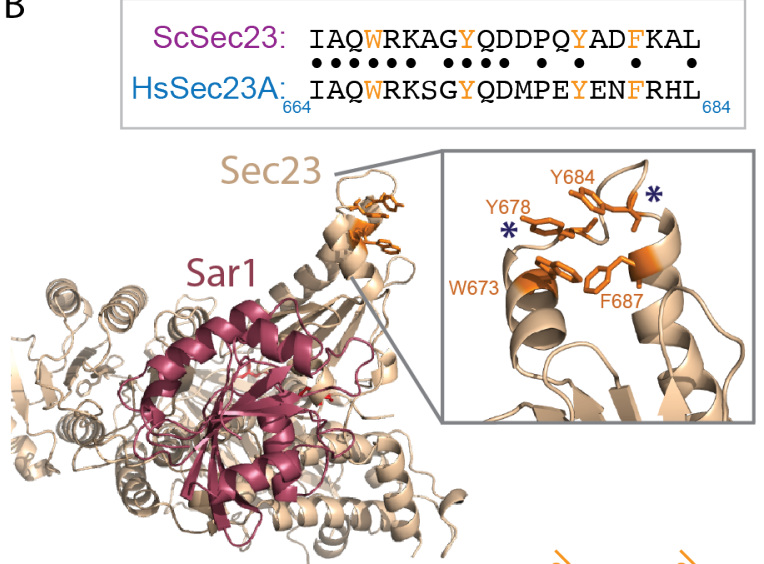

D
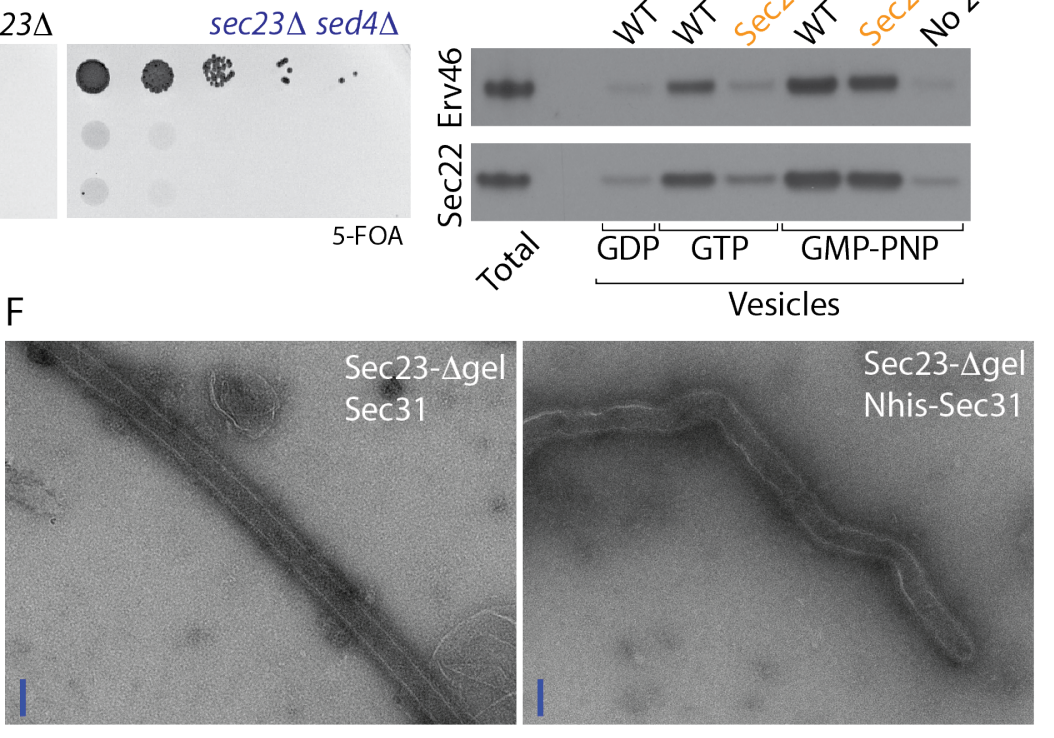

Figure 1. Sec23 gelsolin domain is dispensable for coat assembly but contributes to coat

stability. A. Cartoon depicting vesicle formation from the endoplasmic reticulum (ER) by the

COPII coat, followed by vesicle release, tethering, uncoating and fusion with the Golgi. B.

Structural model (PDB 1M2O) of Sar1 (red) and Sec23 (wheat) highlighting the membranedistal gelsolin domain (inset right) that contains conserved aromatic residues (inset left) that in human Sec23 contribute to PPP binding. C. $\sec 23 \Delta$ and $\sec 23 \Delta \operatorname{sed} 4 \Delta$ strains were transformed with the indicated plasmids and growth tested on 5-FOA, which counterselects for a SEC23::URA plasmid. Growth on 5-FOA is indicative of ability to serve as the sole copy of SEC23. Deletion of the gelsolin loop was tolerated in the $\sec 23 \Delta$ strain but was unable to support viability in the $\sec 23 \Delta$ sed $4 \Delta$ double mutant. D. In vitro budding experiments using yeast microsomal membranes incubated with Sar1, Sec23/Sec24, Sec13/Sec31 and the indicated nucleotides. Vesicle release from the donor membrane is measured by detecting 
incorporation of COPII cargo proteins, Sec22 and Erv46, into a slowly sedimenting vesicle fraction. When wild-type Sec23 was replaced with Sec23- $\Delta$ gel, budding was compromised only in the GTP condition. E. In vitro budding as described for (C) but where one reaction used Sec31 that was tagged with hexahistidine at the $\mathrm{N}$-terminus (lane 2). Sec23- $\Delta$ gel in 5 combination with Nhis-Sec31 was compromised for budding even in the presence of GMPPNP. F. Negative stain electron microscopy of GUVs incubated with Sar1•GMP-PNP and the indicated wild-type or mutant proteins. 


\section{A $\operatorname{Sec} 31$}

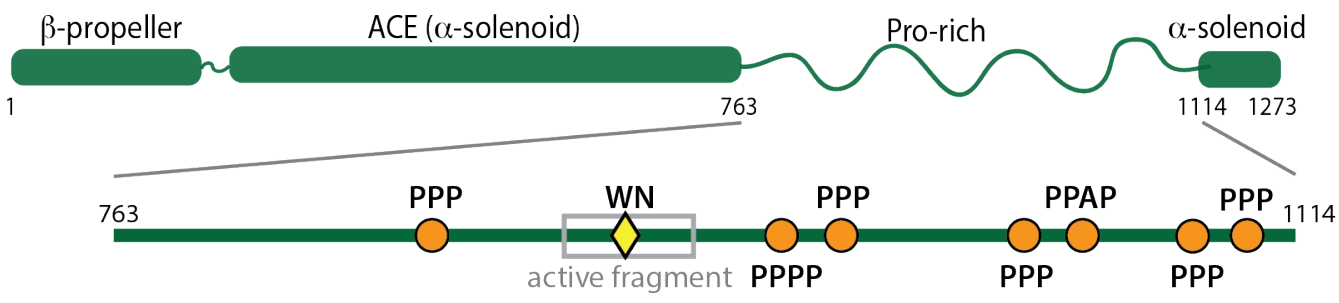

B

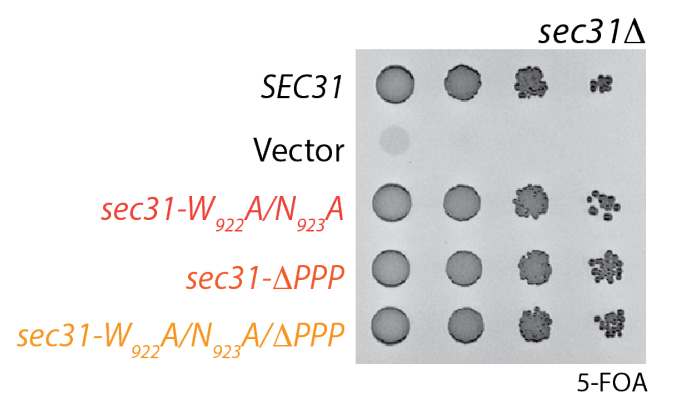

C

D

$\sec 31 \Delta$
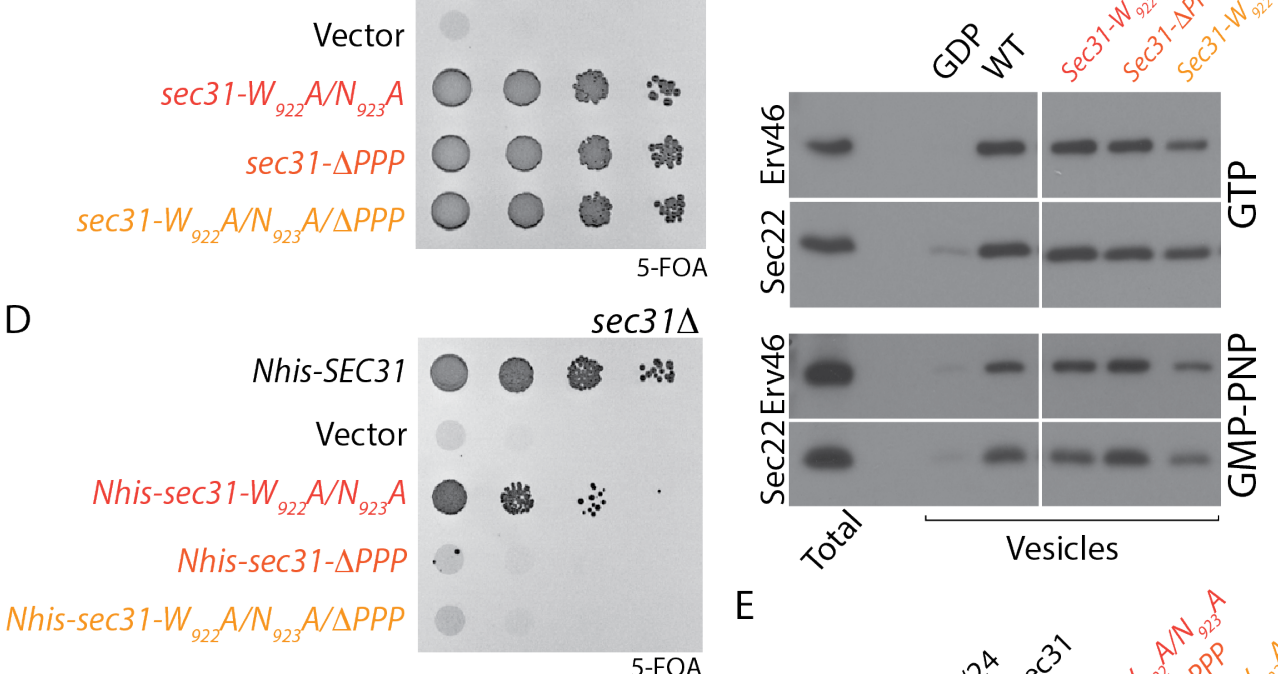

$\mathrm{E}$

$\mathrm{F}$
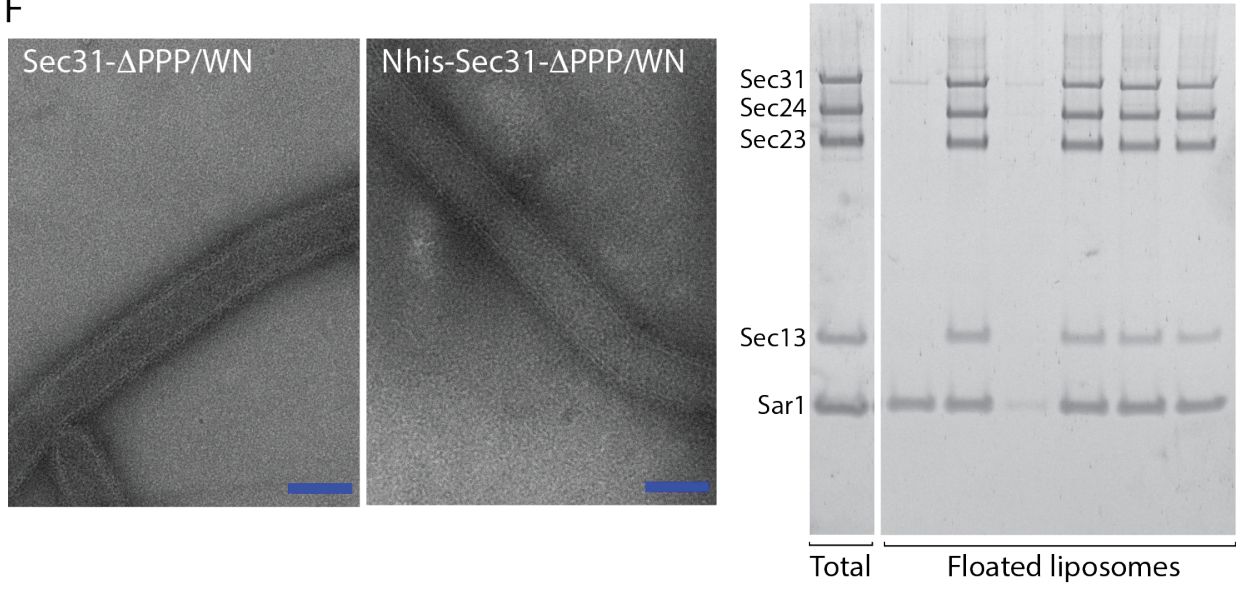

Figure 2. Sec31 known interaction interfaces are individually dispensable for coat

assembly. A. Diagram of Sec31 showing structured elements ( $\beta$-propeller and $\alpha$-solenoids) and the proline-rich disordered region that contains the active fragment (grey box) with

5 catalytic $W_{922} / N_{923}$ residues (yellow diamond) and PPP motifs (orange circles) indicated. B. Serial dilutions of $\sec 31 \Delta$ cells transformed with the plasmids indicated spotted onto 5FOA; mutation of the catalytic residues and/or the PPP motifs had no effect on viability. C. In vitro budding from yeast microsomes with the proteins indicated revealed normal budding 
efficiency, except for the Sec31- $\mathrm{W}_{922} \mathrm{~A} / \mathrm{N}_{923} / \Delta \mathrm{PPP}$ mutant, which was reduced in its ability to generate vesicles. D. Serial dilutions of a sec31 $\Delta$ strain expressing Nhis-Sec31 mutants as indicated were grown on 5FOA, revealing lethality associated with the combination of the $\mathrm{N}$-terminal His tag and loss of PPP motifs. E. Purified COPII proteins (indicated on left in the

5 total fraction) were incubated with synthetic small unilamellar liposomes and floated on a sucrose gradient. Buoyant liposomes and bound proteins were collected and analysed by SDS-PAGE and SYPRO Ruby staining. All mutant protein variants were recruited to liposomes with equal efficiency as the wild-type protein. F. Negative stain electron microscopy of GUVs incubated with the indicated proteins. 


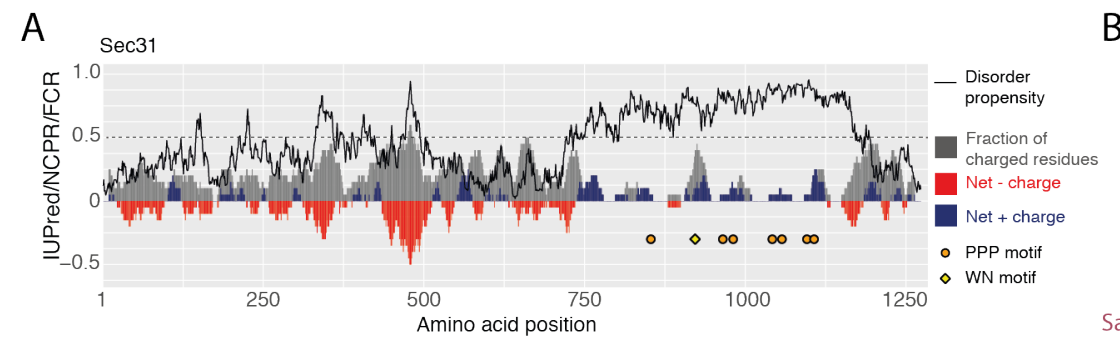

C
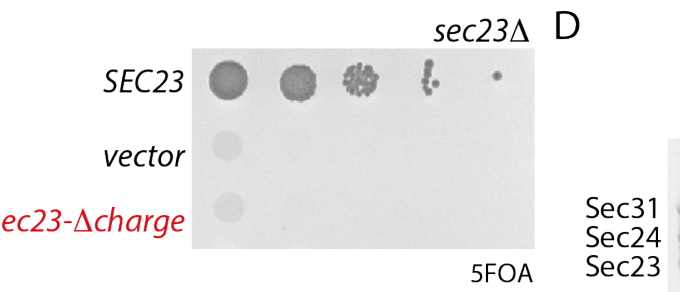

F
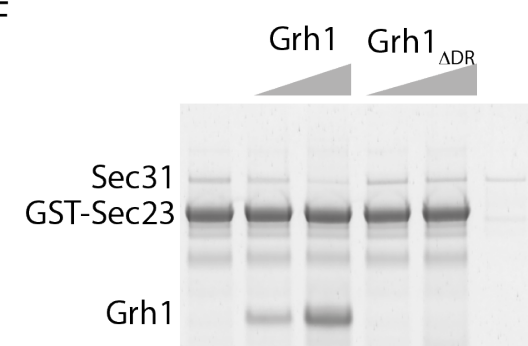

$\operatorname{Sec} 13$

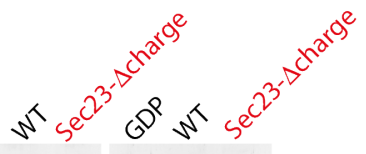

B $\sec 23$

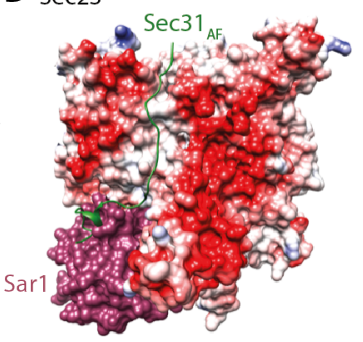

Sec23- $\Delta$ charge
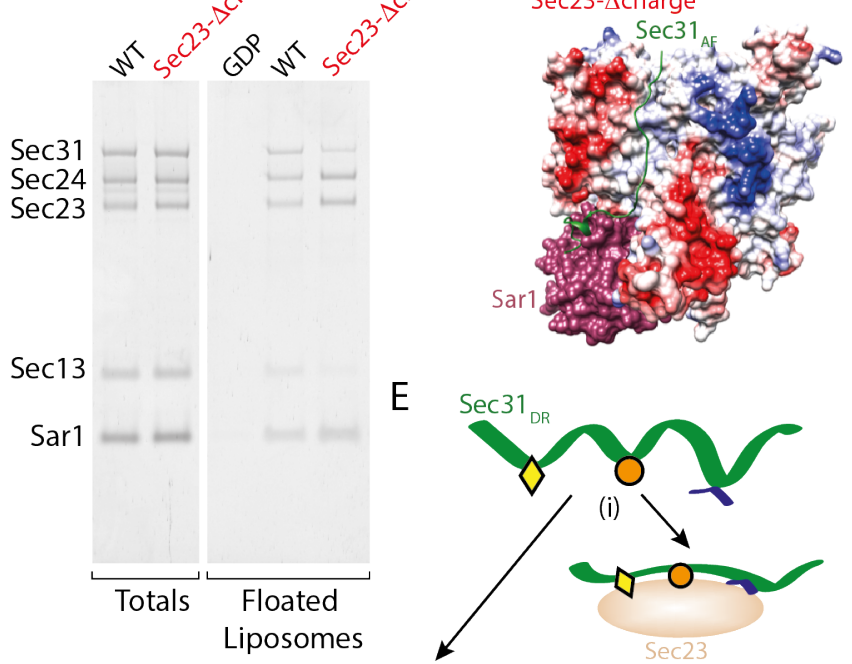

E $\quad \operatorname{Sec} 31_{D R}$

GST

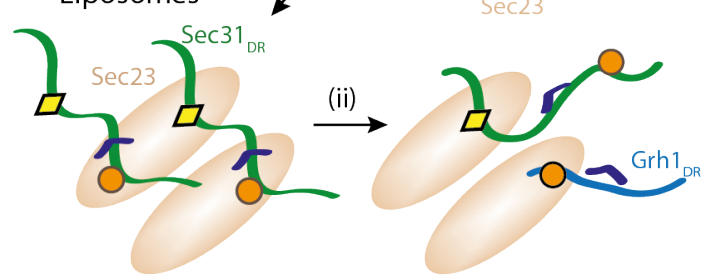

Figure 3. Charge interactions contribute to assembly. A. Charge/disorder plot for Sec31:

the black curve indicates predicted disorder propensity (IUPred); a value $>0.5$ (dashed line) suggests intrinsic disorder. Each grey bar corresponds to fraction of charged residues in a sliding window of 20 amino acids; red/blue bars correspond to net charge per residue in a sliding window of 20 amino acids. PPP motifs are indicated by orange circles, WN motifs are indicated by yellow diamond. B. Surface rendering of the crystal structures of Sar1 (red) and Sec23 (coloured according to electrostatic potential) bound to the active fragment of Sec31 (green), highlighting a negatively charged patch (red region) to the right of the active

10 fragment (left panel), which changes in electrostatic potential in the Sec23- $\Delta$ charge mutant (right panel). C. Serial dilutions of $\sec 23 \Delta$ strains transformed with the plasmids indicated reveals lethality of charge reversal of the negative patch. D. Liposome flotation using the indicated proteins; recruitment of Sec13/Sec31 to liposomes coated with the Sec23- $\Delta$ charge mutant is reduced relative to wild-type. E. Cartoon depicting the three interface elements on the Sec31 disordered region (DR): the $\mathrm{W}_{922} / \mathrm{N}_{923}$ catalytic motif (yellow diamond), PPP 
motifs (orange circle) and charge clusters (blue cap). These elements form a multipartite binding mode such that any individual interaction can be relatively weak but still result in robust binding and oligomerization when present in combination. These elements may bind simultaneously to a single Sec23 molecule (left) or may bridge adjacent Sec23 proteins to 5 promote an inner coat array (right). 
A
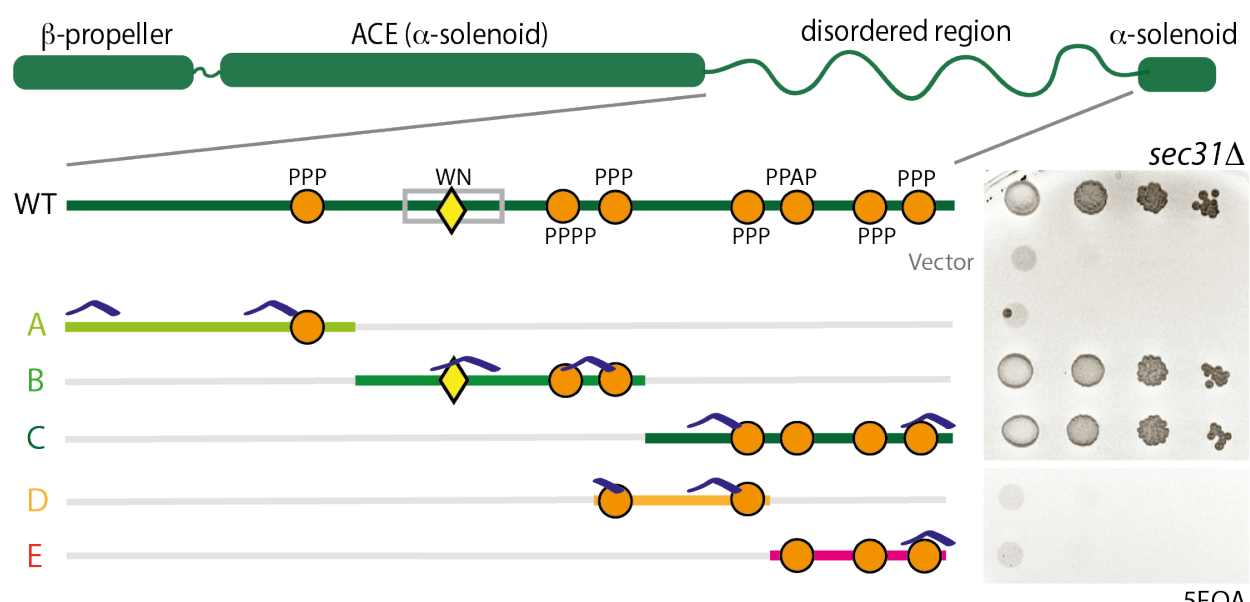

B

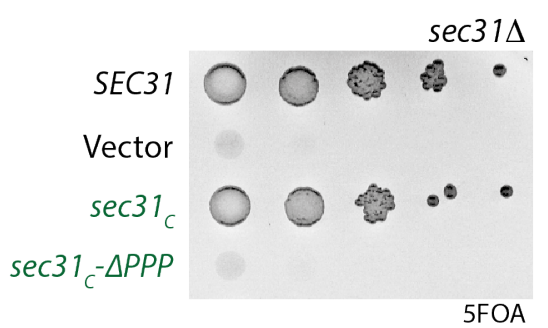

D

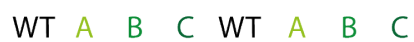

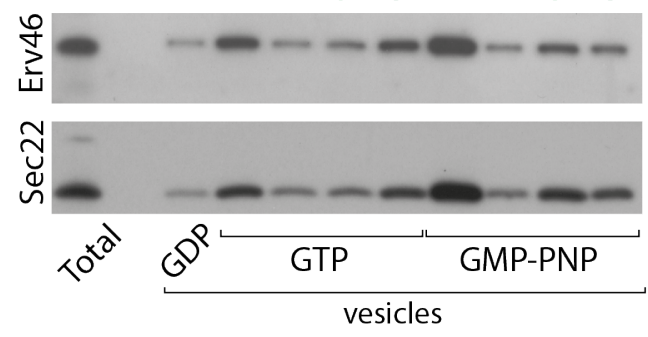

C

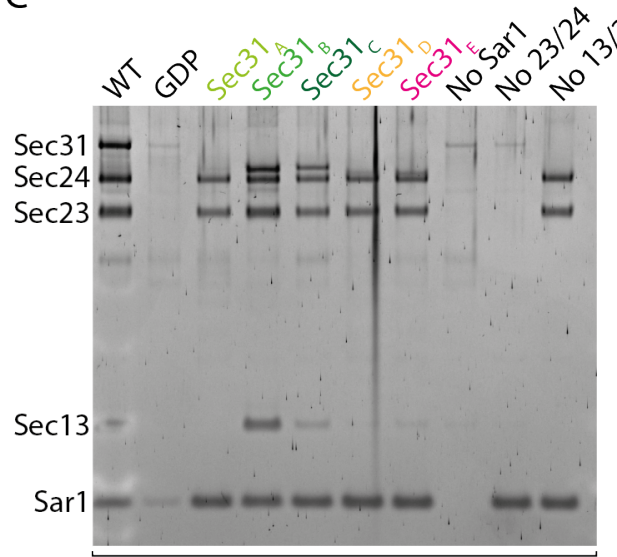

Floated liposomes

Figure 4. Dissection of Sec31 interaction interfaces. A. The disordered region of Sec31 was dissected into the indicated fragments, and the ability of these shortened regions to

function in place of the full disordered region was tested by serial dilution on 5FOA; only the $B$ and $C$ fragments support viability. Features indicated include the $W_{922} / N_{923}$ motif (yellow triangle), PPP motifs (orange circles), and positive charge clusters (blue caps). B. Serial dilutions of Sec31 $\mathrm{C}$ after deletion of PPP motifs reveals the importance of these sequences in the context of a shorter disordered region. C. Recruitment of disordered region fragment proteins to liposomes correlates with viability: only B and C fragments conferred recruitment. D. In vitro budding from microsomal membranes with the indicated proteins also correlated with viability but showed nucleotide dependence. 


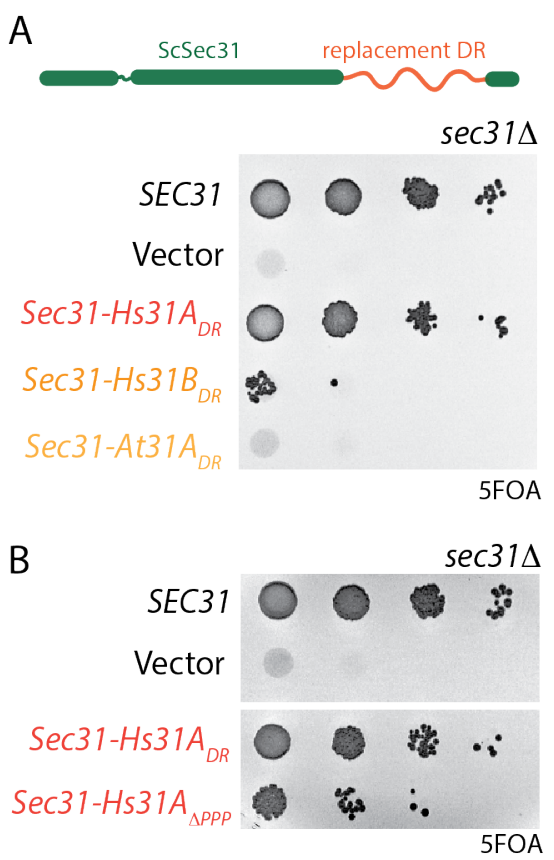

\section{C}
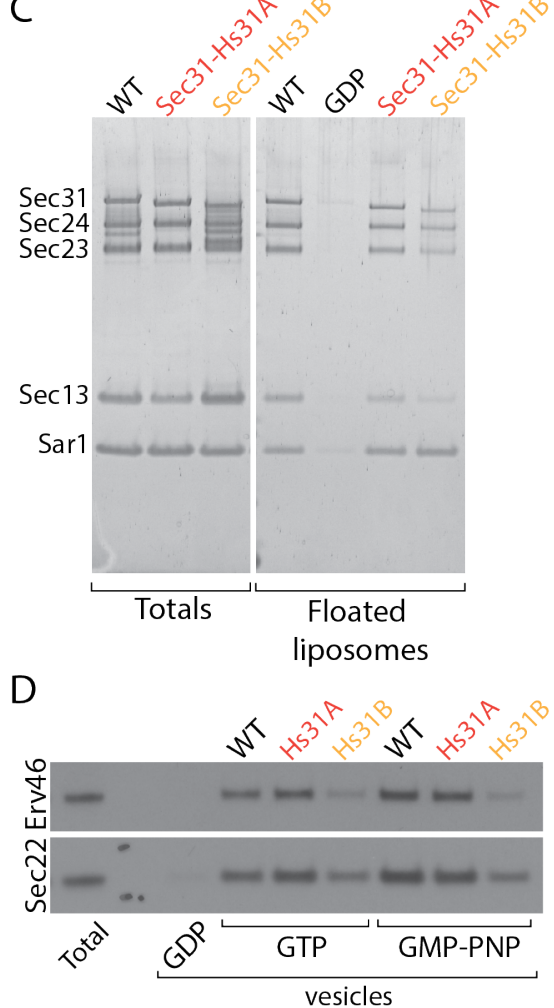

Figure 5. Sec31 disordered region can be functionally replaced with unrelated sequences.

A. Serial dilutions of the indicated constructs where the yeast Sec31 disordered region was replaced with that of other species reveals that only the human Sec31A domain can support viability. B. Serial dilutions of the indicated strains reveals that deletion of the PPP motifs within the human Sec31A disordered region reduces viability. C. Liposome binding experiments with the indicated proteins correlate with viability: coat assembly is driven by the Sec31A region but not by the Sec31B region. D. In vitro microsomal budding experiments using the indicated proteins also correlate with viability: the Sec31A fusion can support vesicle formation with both GTP and GMP-PNP. E. Serial dilutions of chimeric Sec31 constructs containing the indicated unrelated disordered regions reveals that the Las17 domain swap supports viability. 


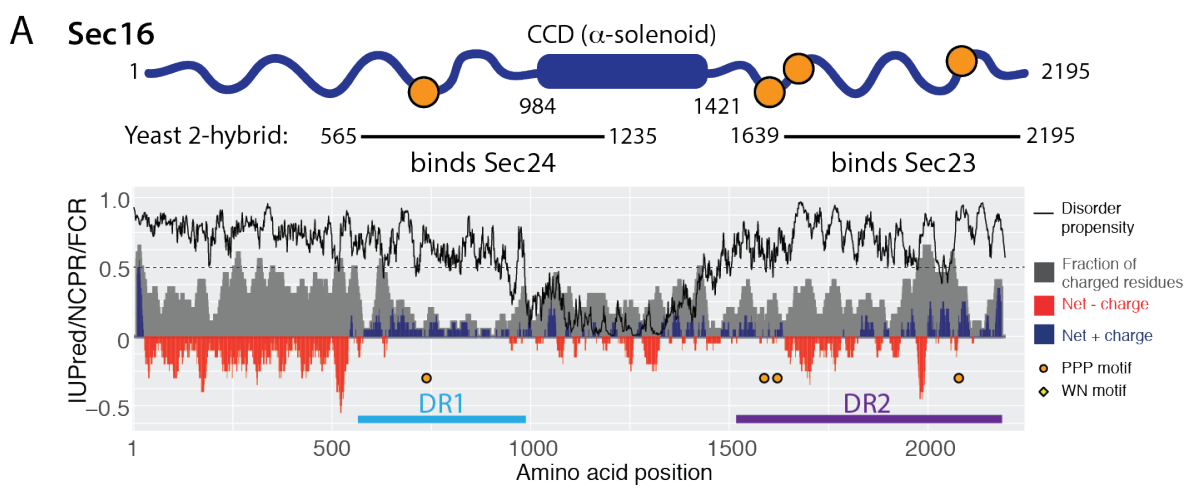

B

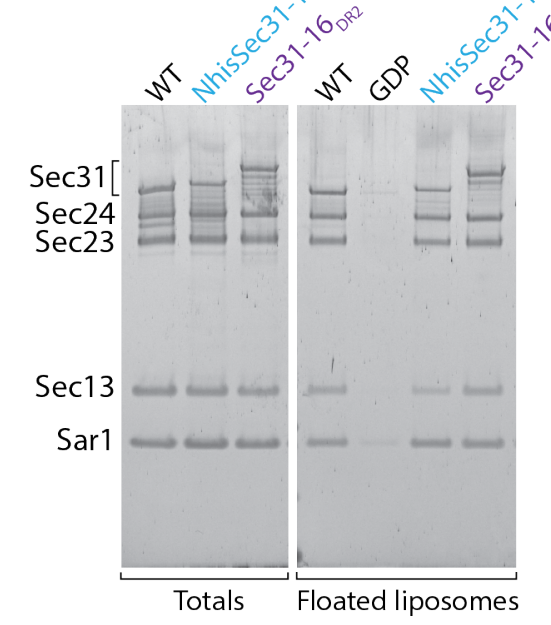

D
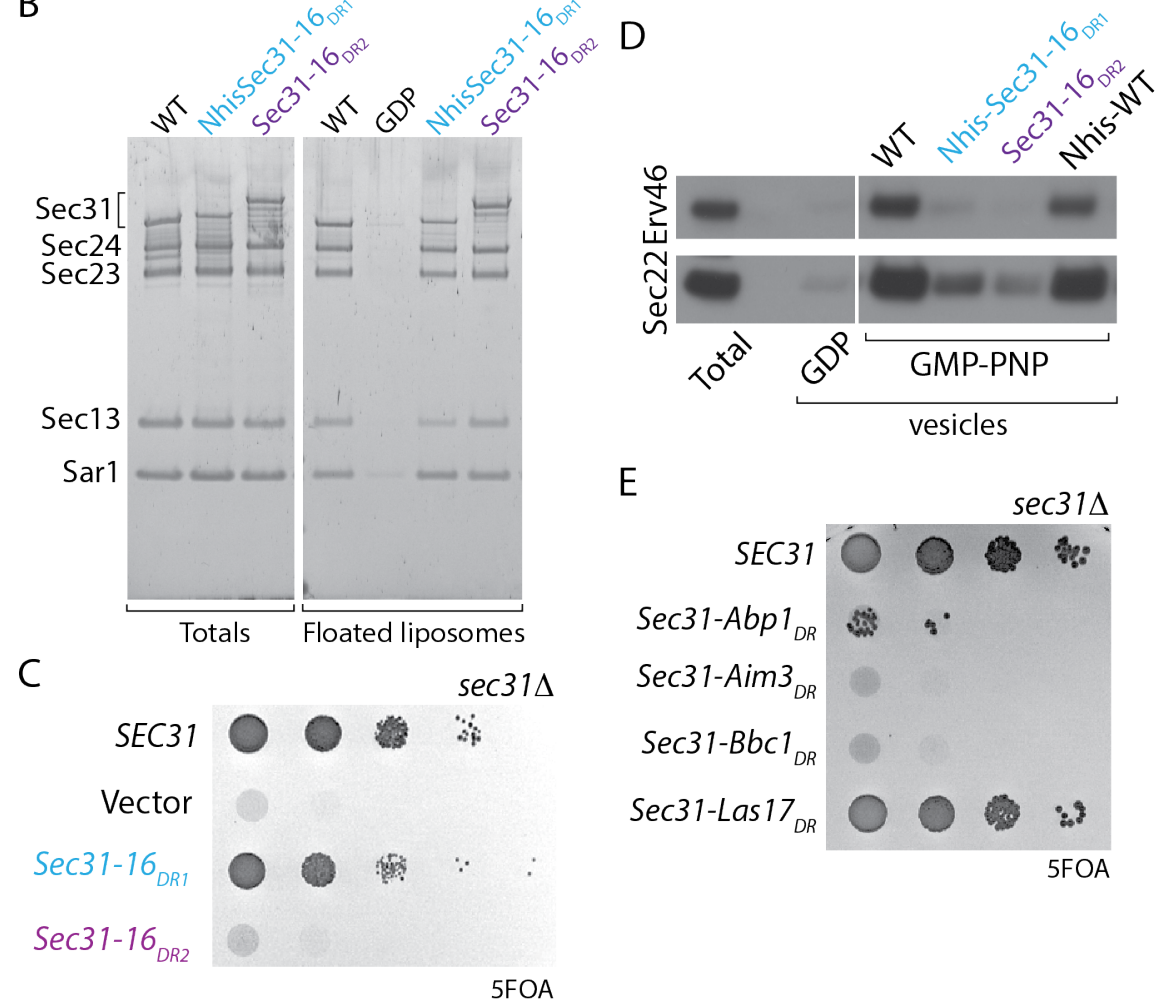

$E$

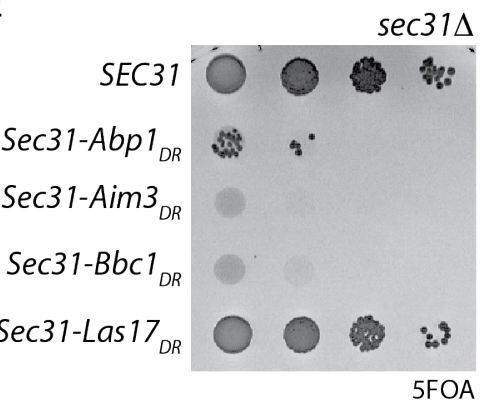

Figure 6. Sec16 disordered regions differentially support coat assembly. A. Diagram and charge-disorder plot of Sec16, highlighting the two disordered regions, DR1 (blue) and DR2 (purple) that interact with Sec24 and Sec23 respectively. Plot is annotated as described in

Figure 3A. B. Serial dilutions of Sec31-Sec16 disordered region chimera reveals that only the first Sec16 disordered region can support viability when replacing the Sec31 DR. C. Liposome binding experiments with the indicated proteins suggest that both Sec16 disordered regions confer coat recruitment. D. In vitro budding from microsomal membranes with the indicated proteins correlates with viability, albeit with reduced efficiency. E. Sec31 can be recruited to immobilized GST-Sec23 (lane 1), but binding is competed by excess Grh1 (lanes 2 and 3 ) but not by truncated Grh1 that lacks its disordered domain (lanes 4 and 5). F. Cartoon depicting dynamic exchange of polymeric Sec31 with a competing low-affinity interaction from the Golgi-localized tether, Grh1. 


\section{Supplementary Materials}

\section{Materials and Methods.}

Strains and plasmids.

Yeast strains used in this study are listed in Table S1. Strains were constructed using standard genetic knock-out and LiAc transformation methods. Cultures were grown at $30^{\circ} \mathrm{C}$ in standard rich medium (YPD: $1 \%$ yeast extract, $2 \%$ peptone, and $2 \%$ glucose) or synthetic complete medium (SC: $0.67 \%$ yeast nitrogen base and $2 \%$ glucose supplemented with amino acids) as required. For testing viability, strains were grown to saturation in SC medium selecting for the mutant plasmid overnight at $30^{\circ} \mathrm{C}$. 10 -fold serial dilutions were made in 96 well trays before spotting onto 5FOA plates ( $1.675 \%$ yeast nitrogen base, $0.08 \%$ CSM, $2 \%$ glucose, $2 \%$ agar, $0.1 \% 5$-fluoroorotic acid). Plates were scanned at day 2 or day 3 after spotting and growth at $30^{\circ} \mathrm{C}$.

Plasmids used in this study are listed in Table S2. Standard cloning methods were used, including PCR amplification of yeast genes from genomic DNA, site-directed mutagenesis using the QuikChange system (Agilent) and Gibson Assembly (New England Biolabs) as per manufacturers' instructions.

Protein expression and purification.

Sar1 was prepared as described (32). Briefly, GST-Sar1 was expressed in bacterial cells by induction for $2 \mathrm{hr}$ with IPTG. Cells were lysed by sonication, and lysates were clarified and incubated with glutathione-agarose beads. The beads were then washed and GST-free Sar1 was generated through thrombin cleavage.

Sec23/Sec24 (Sec23 and His-Sec24) and Sec13/Sec31 (Sec13 and His-Sec31) complexes were co-expressed in Sf9 cells using the pFastbac ${ }^{\mathrm{TM}}$ system. $500 \mathrm{ml}$ of protein-expressing cells were collected and washed with PBS prior to freezing in liquid nitrogen. Cell pellets were lysed with a Dounce homogeniser in cold Lysis Buffer (20mM HEPES pH 8, 250mM sorbitol, 500mM KOAc, $1 \mathrm{mM}$ DTT, $10 \mathrm{mM}$ imidazole, $10 \% \mathrm{v} / \mathrm{v}$ glycerol). Lysates were cleared in JA 25-50 rotor (22 $000 \mathrm{rpm}$, $\left.1 \mathrm{hr}, 4^{\circ} \mathrm{C}\right)$, and the supernatant filtered through a $0.45 \mu \mathrm{m}$ membrane prior to loading onto a HisTrap ${ }^{\text {TM }}$ HP column (GE Healthcare). The following steps were done using the ÄKTA purifier system (GE Healthcare) where Elution buffer was Lysis buffer supplemented with $500 \mathrm{mM}$ imidazole. The column was washed with $4 \%$ and $10 \%$ Elution buffer followed by elution using a linear gradient to $100 \%$ Elution buffer. Peak fractions were checked by SDS-PAGE followed by Coomassie staining. Verified fractions were mixed in a 3:1 ratio with QA buffer (20mM Tris pH 7.5, $1 \mathrm{mM}$ MgOAc, $1 \mathrm{mM}$ DTT, 10\% v/v glycerol) and loaded onto a HiTrap ${ }^{\mathrm{TM}} \mathrm{Q}$ HP column (GE Healthcare). The protein was eluted using a linear salt gradient to a final concentration of $1 \mathrm{M}$ $\mathrm{NaCl}$. Peak fractions were verified using SDS-PAGE and Coomassie staining and flash-frozen in liquid nitrogen. For removal of the 6xHis tag on Sec31, an overnight cleavage with His-TEV was included after the verification of Ni-IMAC fractions. The cleavage was done simultaneously with dialysis into Lysis Buffer. Uncleaved protein and His-TEV were removed by flowing through the HisTrap ${ }^{\text {TM }}$ HP column prior to continuing to the ion-exchange step.

6xHis-Grh1 (and the $\triangle \mathrm{DD}$ variant) were expressed and purified as described above for Sec23/Sec 24 with the following modifications: the lysates were spun in an ultracentrifuge $(100,000 \times \mathrm{g}, 1 \mathrm{hr}$, $4^{\circ} \mathrm{C}$ ). Following the $\mathrm{Ni}-\mathrm{IMAC}$ step the verified fractions were dialysed overnight in $\mathrm{Q}^{*}$ buffer $(20$ 
$\mathrm{mM}$ Tris $\mathrm{pH}$ 7.5, $1 \mathrm{mM}$ MgOAc, $1 \mathrm{mM}$ DTT, $150 \mathrm{mM}$ KOAc, 10\% v/v glycerol). The sample was then concentrated using an sAmicon ${ }^{\circledR}$ Centrifugal Filter Unit (MERCK) and frozen in liquid nitrogen.

In vitro budding from microsomal membranes.

Microsomal membranes were prepared from yeast as described (33). Briefly, yeast cells were grown to mid-log phase in YPD (1\% yeast extract, $2 \%$ peptone, and $2 \%$ glucose), harvested and resuspended in $100 \mathrm{mM}$ Tris $\mathrm{pH} 9.4 / 10 \mathrm{mM}$ DTT to $40 \mathrm{OD}_{600} / \mathrm{ml}$, then incubated at room temperature for $10 \mathrm{~min}$. Cells were collected by centrifugation and resuspended to $40 \mathrm{OD}_{600} / \mathrm{ml}$ in lyticase buffer (0.7M sorbitol, $0.75 \mathrm{X}$ YPD, $10 \mathrm{mM}$ Tris $\mathrm{pH} 7.4,1 \mathrm{mM}$ DTT + lyticase $\left.2 \mu \mathrm{L} / \mathrm{OD}_{600}\right)$, then incubated at $30 \circ \mathrm{C}$ for $30 \mathrm{~min}$ with gentle agitation. Cells were collected by centrifugation, washed once with $2 X$ JR buffer (0.4M sorbitol, 100mM KOAc, 4mM EDTA, 40mM HEPES pH 7.4) at 100 $\mathrm{OD}_{600} / \mathrm{ml}$, then resuspended in $2 \mathrm{XJR}$ buffer at $400 \mathrm{OD}_{600} / \mathrm{ml}$ prior to freezing at -80 으. Spheroplasts were thawed on ice, and an equal volume of ice cold $\mathrm{dH} 20$ added prior to disruption with a motor-driven Potter Elvehjem homogenizer at 4 으. The homogenate was cleared by low speed centrifugation and crude membranes collected by centrifugation of the low-speed supernatant at $27,000 \times \mathrm{g}$. The membrane pellet was resuspended in $~ 6 \mathrm{~mL}$ of buffer $\mathrm{B} 88$ ( $20 \mathrm{mM}$ HEPES pH 6.8, 250mM sorbitol, $\left.150 \mathrm{mM} \mathrm{KOAc,} 5 \mathrm{mM} \mathrm{Mg}(\mathrm{OAc})_{2}\right)$ and loaded onto a step sucrose gradient composed of $1 \mathrm{~mL} 1.5 \mathrm{M}$ sucrose in $\mathrm{B} 88$ and $1 \mathrm{~mL} 1.2 \mathrm{M}$ sucrose in B88. Gradients were subjected to ultracentrifugation at $190,000 \times \mathrm{g}$ for $1 \mathrm{~h}$ at 4 으. Microsomal membranes were collected from the $1.2 \mathrm{M} / 1.5 \mathrm{M}$ sucrose interface, diluted 10 -fold in $\mathrm{B} 88$ and collected by centrifugation at $27,000 \times \mathrm{g}$. The microsomal pellet was resuspended in a small volume of B88 and aliquoted in $1 \mathrm{mg}$ total protein aliquots until use.

Budding reactions were performed as described (6). Briefly, 1mg of microsomal membranes per 68 reactions was washed $3 x$ with $2.5 \mathrm{M}$ urea in $\mathrm{B} 88$ and $3 \mathrm{x}$ with $\mathrm{B} 88$. Budding reactions were set up

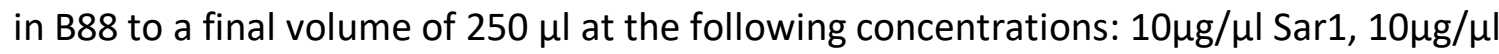
Sec23/Sec24, 20 $\mathrm{g} / \mu \mathrm{l} \mathrm{Sec13/Sec31}$ and 0.1mM nucleotide. Where appropriate, an ATP regeneration mix was included (final concentration $1 \mathrm{mM}$ ATP, 50 $\mu \mathrm{M}$ GDP-mannose, 40mM creatine phosphate, $200 \mu \mathrm{g} / \mathrm{ml}$ creatine phosphokinase). Reactions were incubated for $30 \mathrm{~min}$ at $25^{\circ} \mathrm{C}$ and a $12 \mu \mathrm{l}$ aliquot collected as the total fraction. The vesicle-containing supernatant was collected after pelleting the donor membrane (15 $\left.000 \mathrm{rpm}, 2 \mathrm{~min}, 4^{\circ} \mathrm{C}\right)$. Vesicle fractions were then collected by centrifugation in a Beckman TLA-55 rotor $\left(50000 \mathrm{rpm}, 25 \mathrm{~min}, 4^{\circ} \mathrm{C}\right)$. The supernatant was aspirated, the pelleted vesicles resuspended in SDS sample buffer and heated for $10 \mathrm{~min}$ at $55^{\circ} \mathrm{C}$ with mixing. The samples were then analysed by SDS-PAGE and immunoblotting for Sec22 (Miller lab antibody) and Erv46 (a gift from Charles Barlowe).

In vitro binding to small synthetic liposomes.

Liposome binding experiments were performed as described (6). Briefly, synthetic liposomes of "major/minor" composition (50 mol\% phosphatidylcholine, 21 mol\% phosphatidylethanolamine, 8 mol\% phosphatidylerine, 5 mol\% phosphatidic acid, 9 mol\% phosphatidylinositol, $2.2 \mathrm{~mol} \%$ phosphatidylinositol-4-phosphate, $0.8 \%$ mol\% phosphatidylinositol-4,5-bisphosphate, 2 mol\% cytidine-diphosphate-diacylglycerol, supplemented with 2 mol\% TexasRed-

phosphatidylethanolamine and $20 \%(\mathrm{w} / \mathrm{w}$ ) ergosterol) were dried to a lipid film in a rotary evaporator and rehydrated in HKM buffer (20mM HEPES pH 7.0, $160 \mathrm{mM} \mathrm{KOAc,} 1 \mathrm{mM} \mathrm{MgCl} 2$ ). The lipid suspension was extruded 17 times through a polycarbonate filter of $0.4 \mu \mathrm{m}$ pore size. Purified COPII components and lipids were mixed to final concentrations of $0.27 \mathrm{mM}$ liposomes, $15 \mu \mathrm{g} / \mathrm{ml}$ 
Sar1, 20 $\mathrm{g} / \mathrm{ml} \mathrm{Sec23} / \mathrm{Sec} 24,30 \mu \mathrm{g} / \mathrm{ml} \mathrm{Sec13/Sec31}$ and $0.1 \mathrm{mM}$ nucleotide in $75 \mu \mathrm{l}$ HKM Buffer. Binding reactions were incubated for $30 \mathrm{~min}$ at $25^{\circ} \mathrm{C}$. Each sample was mixed with $50 \mu \mathrm{l} 2.5 \mathrm{M}$ Sucrose-HKM, then $100 \mu \mathrm{L}$ transferred to a ultracentrifuge tube, overlaid with $100 \mu \mathrm{l} 0.75 \mathrm{M}$ Sucrose-HKM and $20 \mu \mathrm{l} \mathrm{HKM}$. The gradients were spun $\left(100000 \mathrm{rpm}, 25 \mathrm{~min}, 24^{\circ} \mathrm{C}\right.$ with slow acceleration/deceleration) in a Beckman TLA-100 rotor. The top $30 \mu \mathrm{l}$ of the gradients were collected and normalised for lipid recovery using Typhoon FLA 7000 scanner (GE). Samples were then resolved by SDS-PAGE and visualised using SYPRO Red staining.

GUV budding and electron microscopy.

GUVs of "major/minor" composition were made by electroformation, as described previously (3). Purified COPII proteins (1 $\mu$ M Sar1, 320nM Sec23/24 and 170nM Sec13/31) were incubated with GUVs (10\% v/v), 1mM GMP-PNP (Sigma) and 2.5mM EDTA in HKM buffer (20 mM HEPES, $50 \mathrm{mM}$ $\mathrm{KOAc}, 1.2 \mathrm{mM} \mathrm{MgCl}$, $\mathrm{pH}$ 6.8) at room temperature for 1-2 hours. Mutants were used in some reactions, as indicated. For negative stain electron microscopy, $4 \mu \mathrm{L}$ of the reconstitution was added to negatively glow-discharged grids (EM Sciences, CF300-Cu) and stained with $2 \%$ uranyl acetate following standard procedures. Grids were viewed with the F20 (Tecnai $200 \mathrm{kV}$ ) electron microscope equipped with a DE20 (Direct Electron, CA) detector. Images were collected as unaligned and summed frames with a dose of 20-30 e\% pixel/second.

\section{GST-Sec23 pulldowns.}

For the competition assays $40 \mu \mathrm{l}$ of $50 \%$ slurry of glutathione Magbeads (GenScript) was precoupled with 100nM GST or GST-Sec23 for $1 \mathrm{hr}$ at $4^{\circ} \mathrm{C}$ in binding buffer (25mM HEPES pH 7.5; $150 \mathrm{mM} \mathrm{NaCl}$; $1 \mathrm{mM}$ DTT; $2 \mathrm{mM}$ EDTA; $0.5 \mathrm{mM} \mathrm{MgCl2;} \%$ v/v Triton X-100; protease inhibitors (Roche)). Beads were washed once with binding buffer and mixed with 500nM Sec13/Sec31 and Grh1 or Grh1- $\triangle \mathrm{DD}$ (at $100 \mathrm{nM}$ or $1 \mathrm{mM}$ ) in a total volume of $150 \mu \mathrm{l}$ and rotated for $2 \mathrm{hr}$ at $4^{\circ} \mathrm{C}$. Beads were washed three times with binding buffer and the samples eluted in $25 \mu \mathrm{l} 3 \mathrm{x}$ SDS Sample Buffer. Samples were heated for $5 \mathrm{~min}$ at $98^{\circ} \mathrm{C}$, separated by SDS-PAGE and stained with SYPRO ruby.

\section{Bioinformatic analyses.}

For analyses of sequence features of disordered domains, sequences of Saccharomyces cerevisiae Sec31, Sec16, Las17, Grh1, as well as human Sec31A, Sec31B, and Arabidopsis Sec31A were collected from Uniprot. The residue specific disorder propensity was calculated using IUPred2A using long disorder mode (34). Charge properties, including fraction of charged residues (FCR) and net charge per residue (NCPR), were calculated using package locaICIDER (35), with a sliding window size of 20. PPP motifs are identified as PPP $(n>2)$, PPAP or PAPP. The analyses were performed in Python. Data were assembled and plotted by R using custom-written scripts. For sequence similarities between disordered domains of Sec31 orthologs, yeast Sec31 (749-1174), human Sec31A (780-1112), human Sec31B (811-1078), and Arabidopsis Sec31A (722-860) were chosen for analysis. The residues were chosen based on cutoff of continuous residues predicted to have high disordered propensity (IUPred $>0.5$ ). Due to the striking difference among the lengths of these domains, three methods of alignment, pairwise local, pairwise global, multiple sequence alignment (MSA), were used to retrieve pairwise sequence similarity between the yeast Sec 31 disordered domain and each of the three homologs. Sequence alignments were performed using EMBOSS water, EMBOSS needle, Clustal Omega respectively, with default parameters (36). 


\section{Supplementary Figures.}

\section{Figure S1}

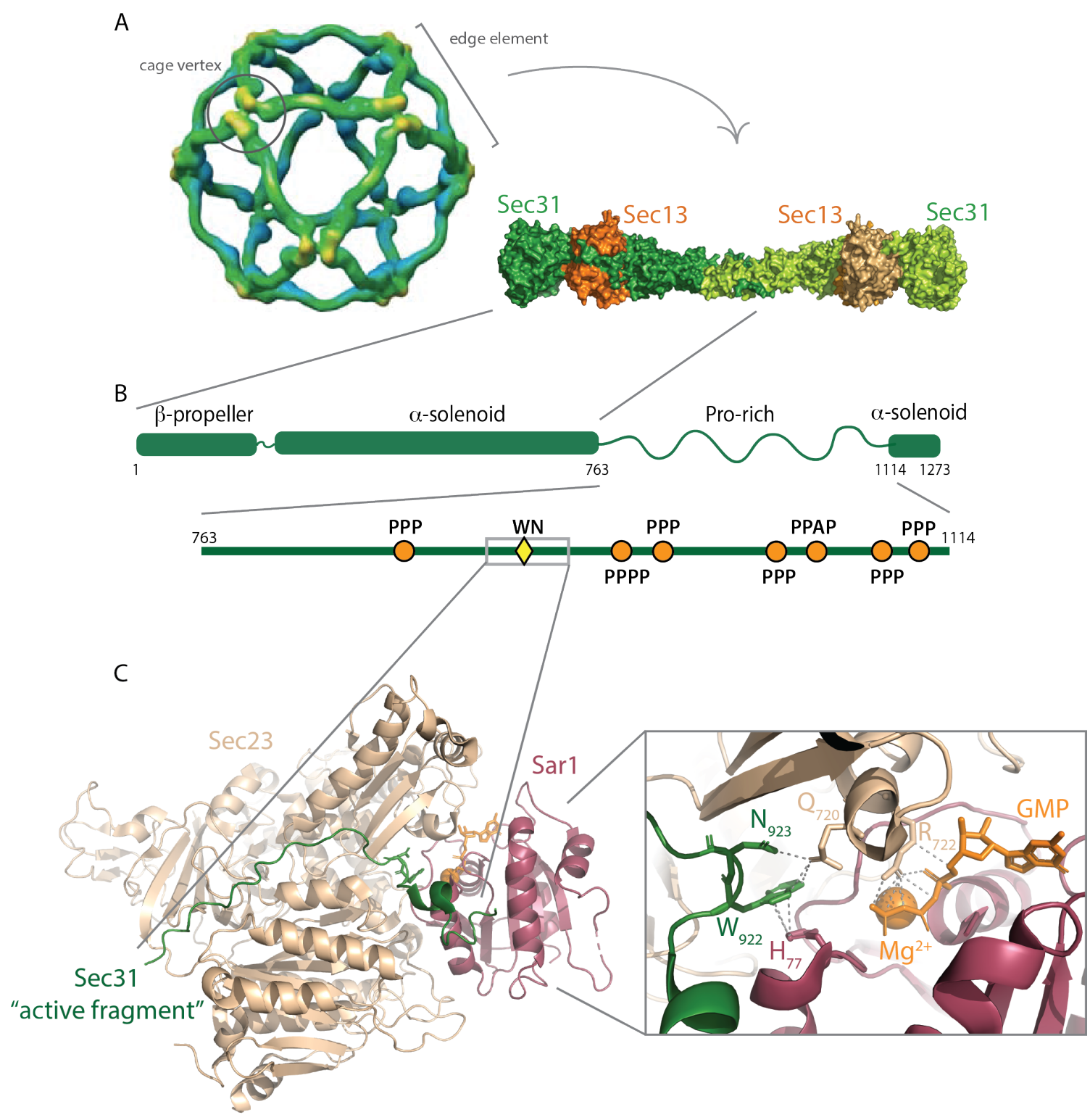

Figure S1. Structure and assembly of the COPII outer coat. A. Cryo-EM model of the cage structure formed by mammalian Sec13/Sec31 (9) showing the "cage vertex" where Sec31 $\beta$ propeller domains tetramerize, and crystal structure (PDB 2PM6 and 2PM9 (8)) of the yeast Sec13/Sec31 "edge element" that forms the assembly unit (8). B. Cartoon of Sec31 showing structured elements ( $\beta$-propeller and $\alpha$-solenoid) and the large proline-rich disordered domain that contacts Sec23/Sar1. Within the Pro-rich domain, several motifs are noted, including tripleproline (PPP) motifs (orange circles), and the "active fragment", which contains the key W922 and $\mathrm{N}_{923}$ residues that stimulate GTP hydrolysis on Sar1 (yellow diamond). C. Crystal structure (PDB 2QTV (11)) of Sar1/Sec23 bound to the active fragment of Sec31 (green), with GTP binding pocket enlarged (right) showing how the catalytic $\mathrm{W} / \mathrm{N}$ residues help position the catalytic $\mathrm{H}_{77}$ and other residues within the GTP binding pocket. 


\section{Figure S2}
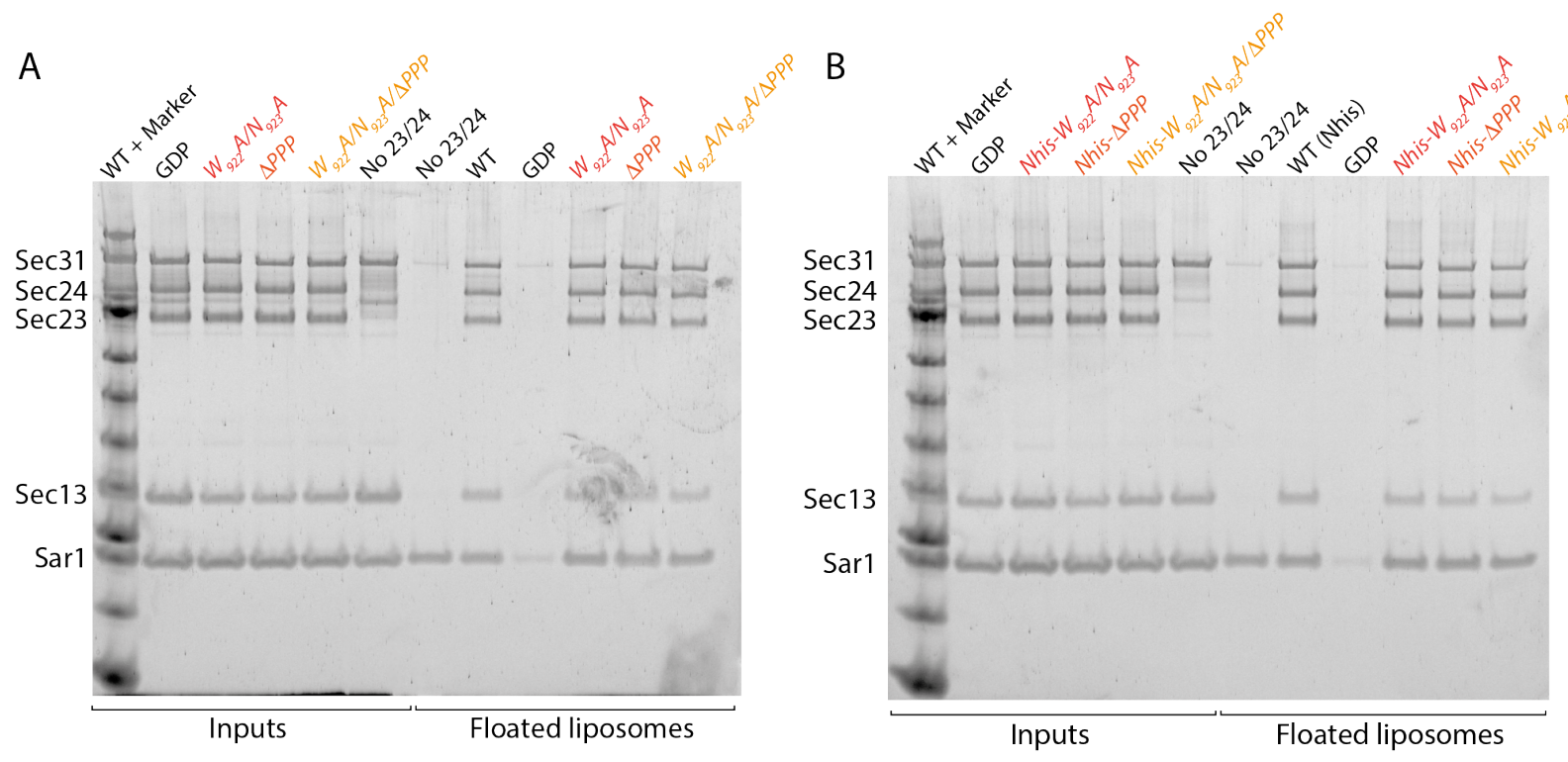

Figure S2. Coat assembly on synthetic liposomes is not perturbed by Sec31 mutations. Purified Sar1, Sec23/Sec24 and Sec13/Sec31 were incubated with synthetic unilamellar liposomes in the presence of GMP-PNP (inputs), and the liposomes purified by flotation through a sucrose gradient. Bound material (floated liposomes) was collected and examined by SDS-PAGE where assembled coat proteins can be distinguished. Mutation of the catalytic residues (W922A/N923A) or PPP motifs $(\triangle P P P)$ did not reduce recruitment into the coat in either the absence $(A)$ or presence $(B)$ of an additional $\mathrm{N}$-terminal hexahistidine tag on Sec31. 


\section{Figure S3}

A
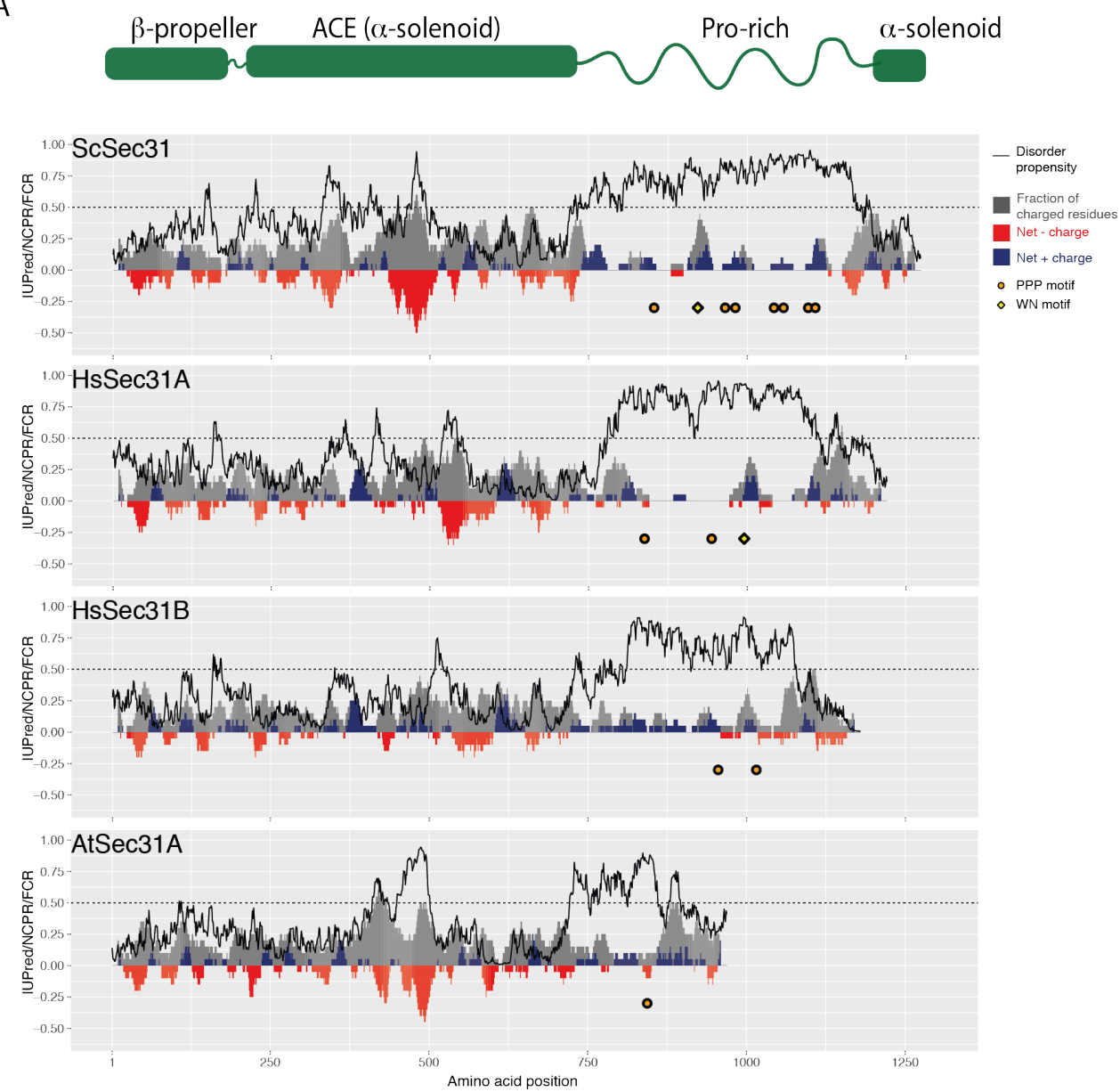

B

\begin{tabular}{cccc}
\hline Similarity (\%) & Pairwise Local & Pairwise Global & MSA \\
\hline HsSec31A & 32.2 & 23.3 & 26.4 \\
HsSec31B & 34.4 & 26.3 & 19.8 \\
AtSec31A & 35.5 & 15.3 & 32.0 \\
\hline
\end{tabular}

Figure S3. Sec31 disordered domains share several features across species. A. Charge-disorder plots for Sec31 orthologs from Saccharomyces cerevisiae (ScSec31), human (HsSec31A and $\mathrm{HsSec} 31 \mathrm{~B}$ ) and Arabidopsis thaliana (AtSec31A). The black curve indicates predicted disorder propensity as calculated by IUPred. A value of IUPred $>0.5$ (dashed line) suggests a strong propensity for being intrinsically disordered. Each grey bar corresponds to fraction of charged residues (FCR) in a sliding window of 20 amino acids, centered at the residue indicated. Red/blue bars at each position correspond to net charge per residue (NCPR) in a sliding window of 20 amino acids. PPP motifs are indicated by orange circles, WN motifs are indicated by yellow diamond. Human Sec31A is the only ortholog that has a disordered domain containing three features: a WN motif, multiple PPP motifs, multiple positively charged clusters. B. Sequence similarity between ScSec31 disordered domains and corresponding domains in orthologs. Three methods of alignment have been used (see methods). None of the orthologs share significant similarity to ScSec31, either globally or locally. 
Figure S4

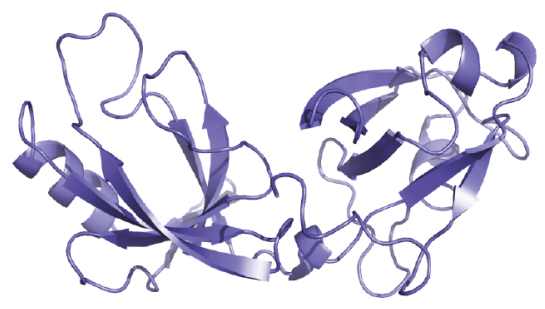

$\alpha$-helix GRASP-homology domain (PDZ)

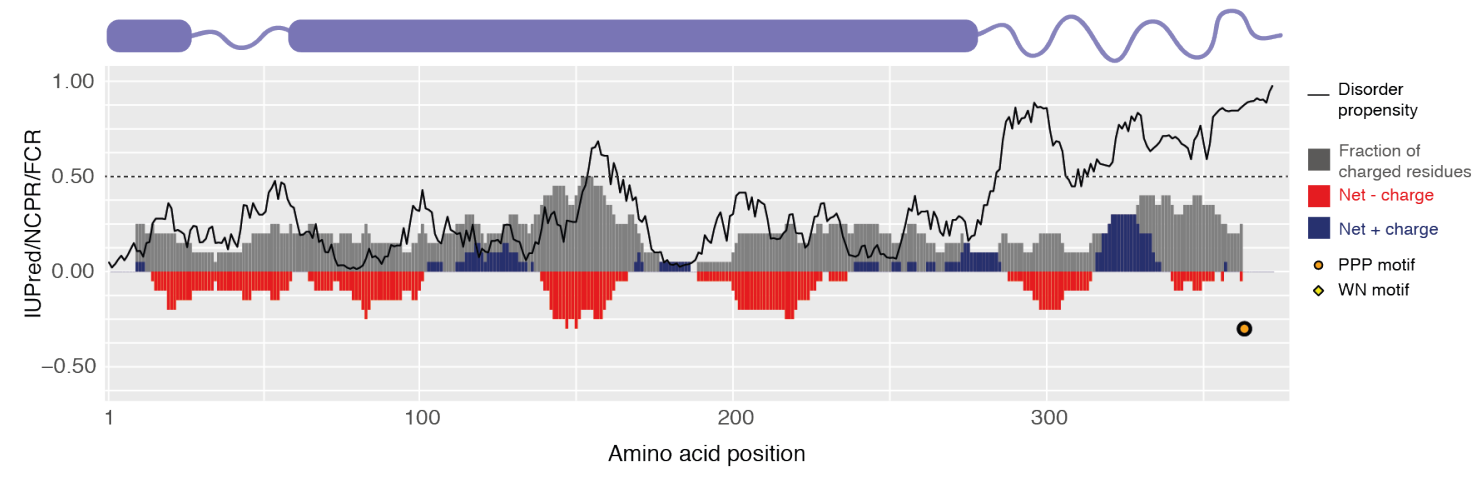

Figure S4. Grh1 contains a disordered domain with PPP motifs. Diagrams of Grh1 structure (blue) showing structural domains ( $N$-terminal $\alpha$-helix, and a PDZ-like GRASP homology domain), and charge-disorder along the polypeptide as described for Figure S3. The C-terminus of Grh1 is predicted to be intrinsically disordered, with a large positively charged cluster and a PPP motif. 
Figure S5

A

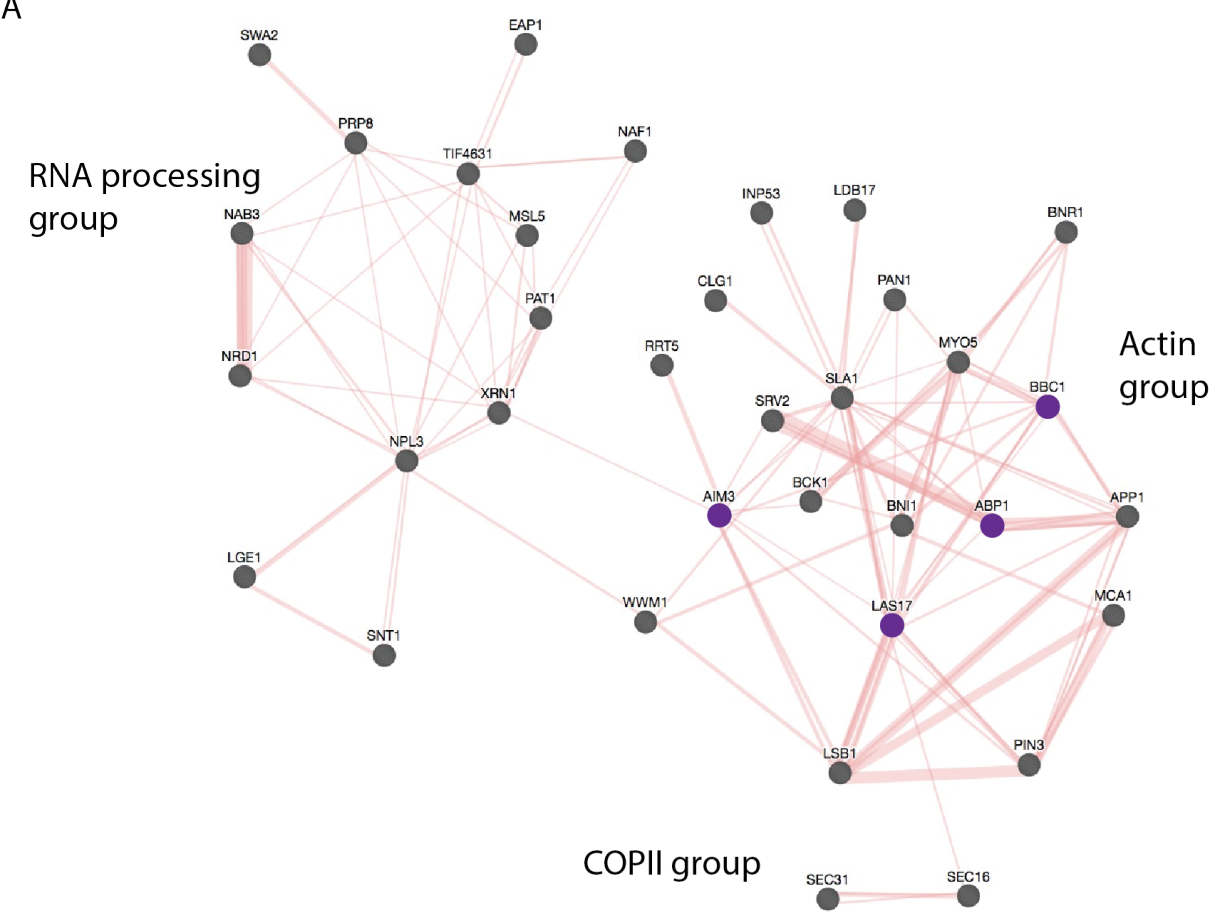

B
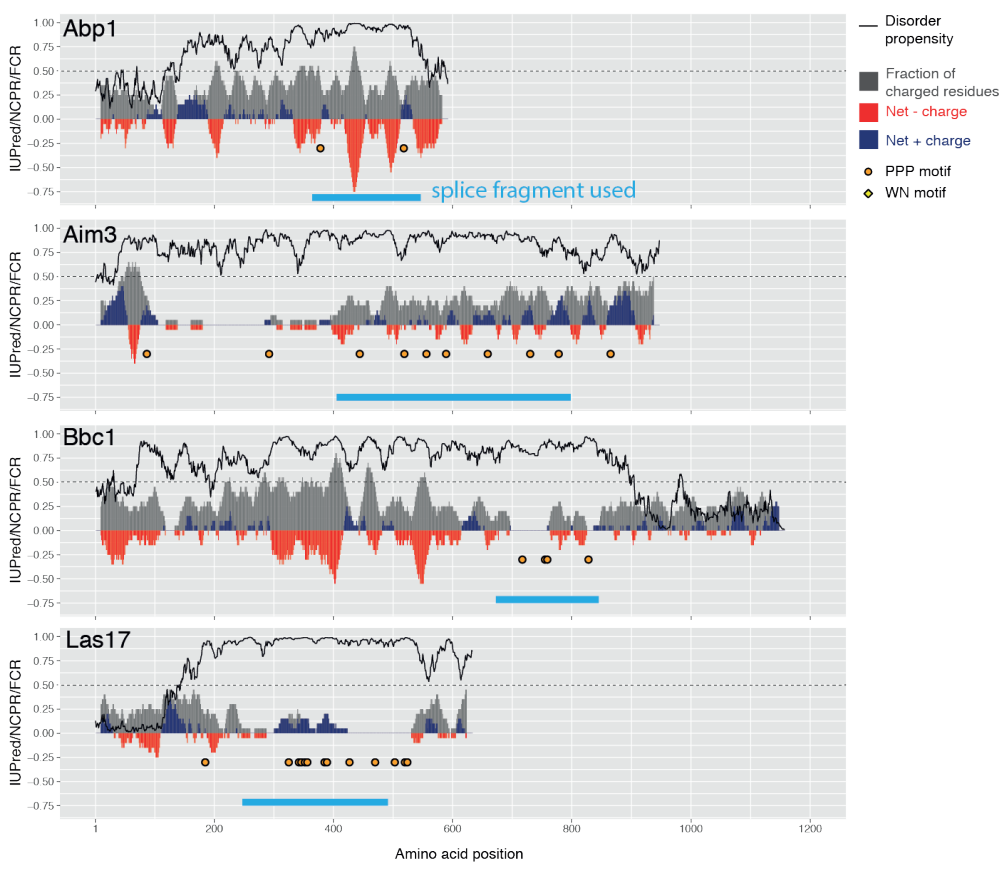

Figure S5. Disordered domains can be found in cytosolic proteins from multiple pathways. A. Protein interaction network diagram of cytosolic proteins that contain intrinsically disordered domains with multiple PPP motifs. Three functional groups are apparent: the COPII coat with Sec31 and Sec16; an endocytosis/actin module; and an RNA processing module. B. Charge/disorder plots for a subset of proteins identified in (A) and selected for testing as replacements for the Sec31 disordered domain. Fragments selected for splicing into Sec31 are indicated by the blue bars. 
Tables.

Table S1 - Yeast strains

\begin{tabular}{|c|c|c|}
\hline Strain & Genotype & Source \\
\hline LMY570 & sec23::HIS ade2-101oc leu2- $\triangle 1$ lys2-801am trp1- $\triangle 63+[p R S 416:: S E C 23-U R A 3]$ & Miller Lab \\
\hline MY868 & sec23::HIS sed4::KANMX ade2-101oc leu2- $\triangle 1$ lys2-801am trp1- $\triangle 63+[p R S 416:: S E C 23-U R A 3]$ & Miller Lab \\
\hline LMY1249 & sec31::NAT pep4::TRP ade2-1 his3-11 leu2-3,112 + [pYCp50::SEC31-URA3] & (3) \\
\hline
\end{tabular}

\section{Table S2 - Plasmids}

\begin{tabular}{|c|c|c|}
\hline Name & Description & Source \\
\hline RSB600 & pRS313 (HIS3, CEN) & $(37)$ \\
\hline RSB602 & pRS314 (TRP1, CEN) & $(37)$ \\
\hline RSB604 & pRS315 (LEU2, CEN) & $(37)$ \\
\hline RSB606 & pRS316 (URA3, CEN) & $(37)$ \\
\hline pLK140 & pRS314 (TRP1::LEU2) SEC23 under native promoter and CYC1 terminator & Miller Lab \\
\hline VSB44 & sec23- $\Delta$ gel (Y678-Y684 replaced with SGS) mutation in pLK140 & This study \\
\hline VSB81 & sec23-4charge (D2R/E4R/D8R/D44R/E45R/E392R/E393R/E526R) mutation in pLK140 & This study \\
\hline pAC20 & pRS316(URA3::LEU2) genomic fragment of SEC31 with 6xHis encoding sequence inserted after M1 & Miller Lab \\
\hline VSB50 & sec31-W922A/N923A mutation in pAC20 & This study \\
\hline VSB69 & $\begin{array}{l}\text { sec31- } \triangle P P P \text { (PPP853-855SGS/PPPP965-968GSGS/PPP981-983GSG/APPP1041- } \\
\text { 1044GSGS/PPAP1057-1060GSGS/PPP1096-1098GSG/PPP1107-1109SGS) mutation in pAC20 }\end{array}$ & This study \\
\hline VSB87 & sec31-W922A/N923A mutation in VSB69 & This study \\
\hline VSB49 & $\begin{array}{l}\text { pRS313 (HIS3, CEN) SEC31 with native } 500 \text { bp upstream and downstream of gene cloned in } \\
\text { BamHI/Notl }\end{array}$ & This study \\
\hline VSB88 & sec31-W922A/N923A mutation in VSB49 & This study \\
\hline VSB58 & $\begin{array}{l}\text { sec31- } \triangle P P P \text { (PPP853-855SGS/PPPP965-968GSGS/PPP981-983GSG/APPP1041- } \\
\text { 1044GSGS/PPAP1057-1060GSGS/PPP1096-1098GSG/PPP1107-1109SGS) mutation in VSB49 }\end{array}$ & This study \\
\hline VSB59 & sec31-W922A/N923A mutation in VSB58 & This study \\
\hline VSB60 & $\sec 31 A(\Delta \mathrm{I} 881-\mathrm{K} 1114)$ mutation in VSB49 & This study \\
\hline VSB61 & sec31B ( $\Delta$ T764-P880/DE998-K1114) mutation in VSB49 & This study \\
\hline VSB62 & $\sec 31 C(\Delta T 764-S 997)$ mutation in VSB49 & This study \\
\hline VSB63 & $\sec 31 D(\Delta \mathrm{T} 764-\mathrm{S} 974 / \Delta \mathrm{P} 1051-\mathrm{K} 1114)$ mutation in VSB49 & This study \\
\hline VSB64 & $\sec 31 E(\Delta T 764-T 1050)$ mutation in VSB49 & This study \\
\hline VSB89 & $\begin{array}{l}\text { sec31C- } \triangle P P P(A P P P 1041-1044 G S G S / P P A P 1057-1060 G S G S / P P P 1096-1098 G S G / P P P 1107-1109 S G S) \\
\text { mutation in VSB62 }\end{array}$ & This study \\
\hline VSB70 & sec31-Hs31ADD (T764-K1114 replaced with HsSec31A P800-P1091) mutation in VSB49 & This study \\
\hline VSB71 & sec31-Hs31BDD (T764-K1114 replaced with HsSec31B S793-E1053) mutation in VSB49 & This study \\
\hline VSB72 & sec31-At31ADD (T764-K1114 replaced with AtSec31A A719-L870) mutation in VSB49 & This study \\
\hline VSB90 & $\begin{array}{l}\text { sec31-Hs31ADD- } \triangle P P P \text { (PPPP802-805SGSG/APP864-866GSG/PPPP908-911SGSG/APP924- } \\
\text { 926GSG/PP934-935GS) mutation in VSB69 }\end{array}$ & This study \\
\hline VSB73 & sec31-Abp1DD (T764-K1114 replaced with Abp1 G361-E528) mutation in VSB49 & This study \\
\hline VSB74 & sec31-Aim3DD (T764-K1114 replaced with Aim3 1401-N788 mutation in VSB49 & This study \\
\hline VSB75 & sec31-Bbc1DD (T764-K1114 replaced with Bbc1 S670-S843) mutation in VSB49 & This study \\
\hline VSB76 & sec31-Las17DD (T764-K1114 replaced with Las17 I306-P529) mutation in VSB49 & This study \\
\hline VSB82 & sec31-16DD1 (T764-K1114 replaced with Sec16 G565-D984) mutation in VSB49 & This study \\
\hline VSB83 & sec31-16DD2 (T764-K1114 replaced with Sec16 Q1513-Q2195) mutation in VSB49 & This study \\
\hline pFB23 & pFastBacHTb-Sec23p & Zanetti Lab \\
\hline pFB24N & pFastBacHTb-Sec24p-NHis & Zanetti Lab \\
\hline pFB13 & pFastBacHTb-Sec13p & Zanetti Lab \\
\hline pFB31N & pFastBacHTb-Sec31p-NHis & Zanetti Lab \\
\hline VSB95 & sec23- $\Delta$ gel (Y678-Y684 replaced with SGS) mutation in pFB23 & This study \\
\hline VSB96 & sec23- $\Delta$ charge (D2R/E4R/D8R/D44R/E45R/E392R/E393R/E526R) mutation in pFB23 & This study \\
\hline VSB97 & sec31-W922A/N923A mutation in pFB31N & This study \\
\hline
\end{tabular}




\begin{tabular}{|c|c|c|}
\hline VSB98 & $\begin{array}{l}\text { sec31- } \triangle P P P \text { (PPP853-855SGS/PPPP965-968GSGS/PPP981-983GSG/APPP1041- } \\
\text { 1044GSGS/PPAP1057-1060GSGS/PPP1096-1098GSG/PPP1107-1109SGS) mutation in pFB31N }\end{array}$ & This study \\
\hline VSB99 & sec31-W922A/N923A mutation in VSB98 & This study \\
\hline VSB100 & $\sec 31 A(\Delta \mid 881-\mathrm{K} 1114)$ mutation in $\mathrm{pFB31N}$ & This study \\
\hline VSB101 & $\operatorname{sec31B}(\Delta \mathrm{T} 764-\mathrm{P} 880 / \Delta \mathrm{E} 998-\mathrm{K} 1114)$ mutation in pFB31N & This study \\
\hline VSB102 & $\sec 31 C(\Delta T 764-S 997)$ mutation in $\mathrm{pFB31N}$ & This study \\
\hline VSB103 & $\sec 31 D(\Delta \mathrm{T} 764-\mathrm{S} 974 / \Delta \mathrm{P} 1051-\mathrm{K} 1114)$ mutation in $\mathrm{pFB} 31 \mathrm{~N}$ & This study \\
\hline VSB104 & $\operatorname{sec31E}(\Delta \mathrm{T} 764-\mathrm{T} 1050)$ mutation in $\mathrm{pFB31N}$ & This study \\
\hline VSB105 & sec31-Hs31ADD (T764-K1114 replaced with HsSec31A P800-P1091) mutation in pFB31N & This study \\
\hline VSB106 & sec31-Hs31BDD (T764-K1114 replaced with HsSec31B S793-E1053) mutation in pFB31N & This study \\
\hline VSB107 & sec31-16DD1 (T764-K1114 replaced with Sec16 G565-D984) mutation in pFB31N & This study \\
\hline VSB108 & sec31-16DD2 (T764-K1114 replaced with Sec16 Q1513-Q2195) mutation in pFB31N & This study \\
\hline VSB85 & pFastBacHTB GRH1 & This study \\
\hline VSB109 & pFastBacHTB GRH1- $\triangle D D(\triangle \mathrm{A} 318-\mathrm{D} 372)$ & This study \\
\hline
\end{tabular}

